\title{
The origin of the high stability of 3 '-terminal uridine tetrads: contributions of hydrogen bonding, stacking interactions, and steric factors evaluated using modified oligonucleotide analogs
}

\author{
WITOLD ANDRAKOJĆ, KAROL PASTERNAK, JOANNA SARZYŃSKA, KAROLINA ZIELIŃSKA, \\ RYSZARD KIERZEK, and ZOFIA GDANIEC
}

Institute of Bioorganic Chemistry, Polish Academy of Sciences, 61-704 Poznan, Poland

\begin{abstract}
RNA G-quadruplexes fold almost exclusively into parallel-stranded structures and thus display much less structural diversity than their DNA counterparts. However, also among RNA G-quadruplexes peculiar structural elements can be found which are capable of reshaping the physico-chemical properties of the folded structure. A striking example is provided by a uridine tetrad (U-tetrad) placed on the $3^{\prime}$-terminus of the tetramolecular G-quadruplex. In this context, the U-tetrad adopts a unique conformation involving chain reversal and is responsible for a tremendous stabilization of the G-quadruplex $\left(\Delta \mathrm{T}_{\mathrm{m}}\right.$ up to $\left.30^{\circ} \mathrm{C}\right)$. In this report, we attempt to rationalize the origin of this stabilizing effect by concurrent structural, thermal stability, and molecular dynamics studies of a series of G-quadruplexes with subtle chemical modifications at their 3 '-termini. Our results provide detailed insights into the energetics of the "reversed" U-tetrad motif and the requirements for its formation. They point to the importance of the $2^{\prime} \mathrm{OH}$ to phosphate hydrogen bond and preferential stacking interactions for the formation propensity and stability of the motif.
\end{abstract}

Keywords: G-quadruplex; NMR spectroscopy; U-tetrad; molecular dynamics

\section{INTRODUCTION}

DNA and RNA G-quadruplexes are the most studied and biologically important family of noncanonical nucleic acid architectures (Rhodes and Lipps 2015; Kwok and Merrick 2017; Neidle 2017). Even though all G-quadruplex structures contain a core of planarly stacked G-tetrads stabilized by coordinated monovalent cations, it turns out that this general construction scheme can theoretically be realized by a multitude of different chain conformations (Webba da Silva 2007). Indeed, DNA G-quadruplexes have been experimentally demonstrated to have an astonishing structural heterogeneity that makes the task of a priori predicting a DNA quadruplex topology based on its primary sequence nearly impossible (Dvorkin et al. 2018). On the other hand, RNA G-quadruplexes have been found to fold almost exclusively into parallel-stranded structures (Mendoza et al. 2015; Xiao et al. 2018). This marked difference can be ascribed to the strong aversion of the rG res-

Corresponding authors: zgdan@ibch.poznan.pl, wandralojc@ ibch.poznan.pl

Article is online at http://www.rnajournal.org/cgi/doi/10.1261/rna. 076539.120 . idues to assume the syn conformation around the glycosidic bond, strictly required in non-parallel-stranded G-quadruplex topologies. In consequence, the structural studies of RNA G-quadruplexes have attracted significantly less attention over the years, compared to similar studies of their DNA counterparts. This trend is clearly reflected in the fact that around an order of magnitude less RNA than DNA G-quadruplex structures are deposited in the RCSB PDB (www.rcsb.org) (Berman et al. 2000; Popenda et al. 2019). However, also in the realm of RNA G-quadruplexes, one can find unusual structural building blocks endowing the whole structures with special characteristics. The abovementioned structural uniformity of RNA G-quadruplexes actually makes these special elements even more valuable.

An interesting example of such a special building block are uridine tetrads ( $U$-tetrads) located at the 3 '-termini of

C) 2020 Andrałojć et al. This article is distributed exclusively by the RNA Society for the first 12 months after the full-issue publication date (see http://rnajournal.cshlp.org/site/misc/terms.xhtml). After 12 months, it is available under a Creative Commons License (Attribution-NonCommercial 4.0 International), as described at http://creativecommons. org/licenses/by-nc/4.0/. 
tetramolecular G-quadruplexes. A U-tetrad is formed by four uridine residues and stabilized by only a single hydrogen bond per uridine (as opposed to two hydrogen bonds per guanosine for a G-tetrad) (Cheong and Moore 1992). It may thus come as quite a surprise that when a $U$-tetrad is encountered as the $3^{\prime}$-terminal tetrad of a tetramolecular G-quadruplex then, in this particular structural context, its presence has a profound stabilizing effect on the $\mathrm{G}$-quadruplex fold. The best example is provided by $\mathrm{Xu}$ et al. (2010), where the authors have found that the addition of a U-tetrad at the $3^{\prime}$-terminus of the G-quadruplex formed by a single repeat of the human telomeric sequence leads to an increase of the melting temperature of the G-quadruplex by almost $30^{\circ} \mathrm{C}$. Several more literature examples have been provided in our previous paper (Andrałojć et al. 2019). The stabilizing effect of the $3^{\prime}$-terminal U-tetrad appears even more unusual given that its direct counterpart-the 3 '-terminal T-tetrad-completely lacks this property. This is evident, for example, from the $\sim 30^{\circ} \mathrm{C}$ difference in the melting temperature observed between $r(U G G G U)_{4}$ and $[d(T) r(G G G) d(T)]_{4}$ G-quadruplexes (Zhou et al. 2017).

The available literature contains a substantial amount of structural data concerning $3^{\prime}$-terminal T- and U-tetrads. These data explain the lack of contribution of the $3^{\prime}$-terminal T-tetrad to DNA G-quadruplex stability, as in most known structures the 3'-terminal T-tetrads simply do not form. Instead, the $3^{\prime}$-terminal thymidines appear to be subject to a significant conformational variability (Laughlan et al. 1994; Cáceres et al. 2004; Creze et al. 2007; Lee et al. 2007). On the other hand, for tetramolecular RNA G-quadruplexes bearing 3'-terminal uridines marked dominance of $\mathrm{U}$-tetrad forming conformations is observed, yet still with notable structural diversity present (Deng et al.
2001; Pan et al. 2003a,b,c, 2006a,b; Fyfe et al. 2015). Namely, three distinct modes of U-tetrad formation have been found in the available crystal structures (Fyfe et al. 2015), one with the standard helical backbone conformation and two involving a chain reversal just before the $3^{\prime}$-terminal uridine (for structural details on these conformations see Figure 6 in Fyfe et al. 2015). In several crystals two different conformations have been found to coexist in the unit cell (Deng et al. 2001; Pan et al. 2003a; Fyfe et al. 2015). Thus, the available crystallographic data suggest that 3'-terminal U-tetrads, even though rather energetically favorable, remain structurally labile and exist as a dynamic equilibrium of conformations (Fyfe et al. 2015).

We have recently determined the NMR solution structure of the tetramolecular r(UGGUGGU) 4 quadruplex and found that in solution this molecule possesses a very rigid $3^{\prime}$-terminal region, uniquely adopting a single U-tetrad conformation, featuring a chain reversal prior to the uridine residue (Fig. 1; Andrałojć et al. 2019). The chain reversal is characterized by atypical values of $\alpha$ and $\zeta$ dihedral angles at the $3^{\prime}$-terminal dinucleotide step. Such a conformation is actually very similar to the one encountered most often in crystal structures. Concurrently, the r(UGGUGGU) ${ }_{4}$ was also very thermally stable in $\mathrm{K}^{+}$containing solutions, with the apparent $T_{m}$ of the order of $90^{\circ} \mathrm{C}$. A close-up analysis of the "reversed" U-tetrad motif allowed us to propose links between its presence and the high thermal stability of the G-quadruplex. Namely, the "reversed" U-tetrad at the $3^{\prime}$-termini is stabilized by four (one per RNA strand) $U$ (n)-2'OH to $\mathrm{G}(\mathrm{n}-1)-\mathrm{OP} 2$ hydrogen bonds (Fig. 1). The stability of this hydrogen bonding is best illustrated by the fact that even at $50^{\circ} \mathrm{C}$, the $\mathrm{U}(\mathrm{n})-2^{\prime} \mathrm{OH}$ proton exchanges with the solvent slowly enough to remain observable in the NMR spectrum. Structurally, the formation of this

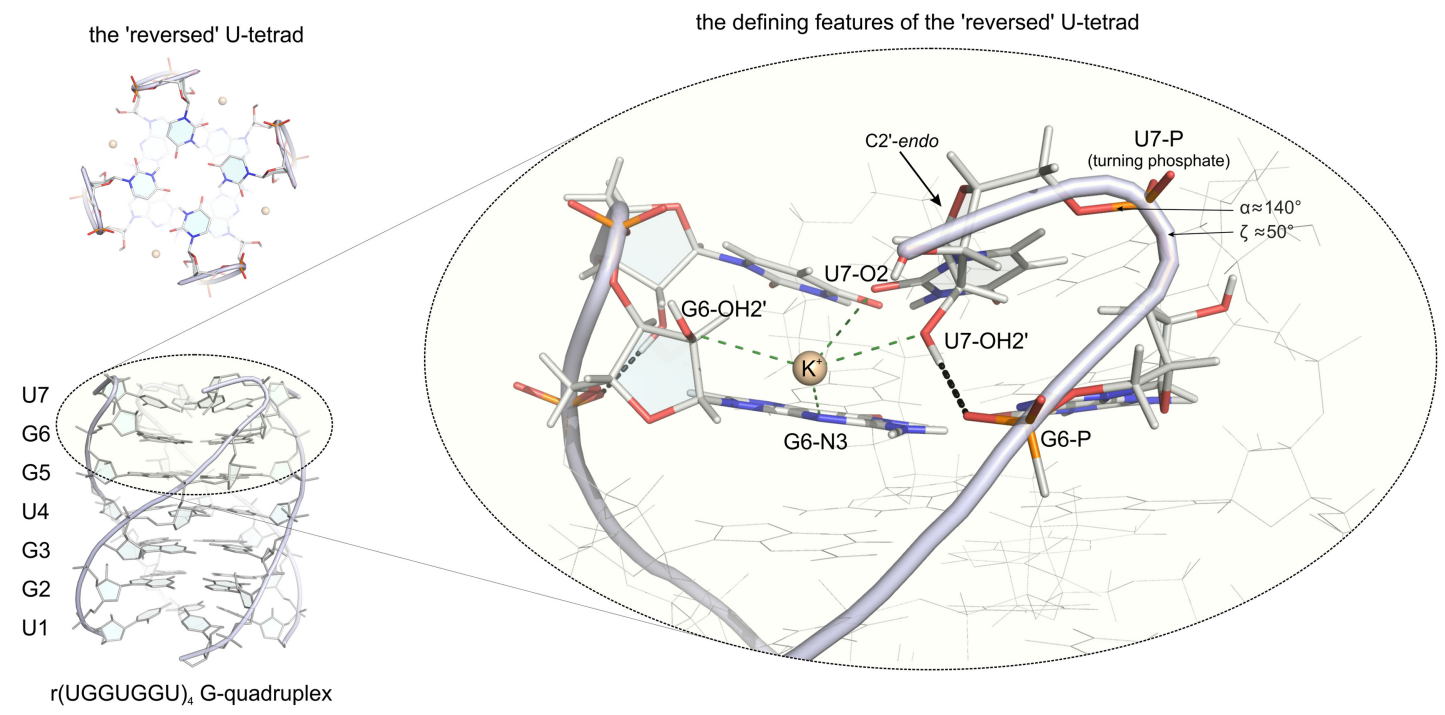

FIGURE 1. The structure of the 3'-terminal "reversed" U-tetrad motif (from PDB ID: 6GE1) and the network of interactions characterizing it. 
hydrogen bond also requires the $3^{\prime}$-terminal residue to assume the C2'-endo sugar pucker (Fig. 1). Moreover, by means of molecular dynamics (MD) simulations we have established the formation of an additional potassium binding site in the quadruplex groove enabled by the presence of the "reversed" U-tetrad. According to the simulations, the binding mode involves direct interaction of the $\mathrm{K}^{+}$ ion with several electronegative atoms in the G-quadruplex (Fig. 1). Both the proposed hydrogen bonding and ion binding are exclusive to RNA (due to the involvement of the $2^{\prime} \mathrm{OH}$ in the interactions), which may partially explain why the closely related T-tetrad element in DNA neither adopts a "reversed" conformation nor induces a particular stabilizing effect.

In the current paper, we revisit the question of the origin of the stabilizing effect of the "reversed" U-tetrad with a different approach. To experimentally verify our previous conclusions, a series of chemically modified analogs of r(UGGUGGU $)_{4}$ was studied. These modifications, introduced at the 3 '-terminal uridine, were designed to either destabilize specific interactions (like the $2^{\prime} \mathrm{OH}$ to phosphate hydrogen bonding, the hydrogen bonding within the tetrad etc.), interfere with selected structural features of the "reversed" U-tetrad (like the C2'-endo sugar pucker of the uridines) or simply induce steric hindrance. For each modification, we analyzed the changes in both the quadruplex stability and its local structure, through a combined NMR, UV and CD analysis. Our analysis demonstrates that all the modifications significantly destabilize the quadruplex fold, highlighting the strict requirement for a nonmodified uridine residue for the stabilizing effect to take place. It also allows us to delve deeper into the energetic basis of the stabilizing effect, among others confirming an important role of the $2^{\prime} \mathrm{OH}$ to phosphate hydrogen bonding and bringing forward the contribution of preferential base stacking.

\section{RESULTS}

\section{Modified G-quadruplex design}

The modified RNA quadruplexes investigated in this study are listed in Table 1 and the sites at which groups of atoms were added, removed, or substituted are graphically presented in Figure 2 and in its expanded version Supplemental Figure S1. The most subtle modification involves substitution of the sugar of U7 with its deoxy- counterpart, deoxyuridine (M1). This modification by itself should be structurally neutral, as the ribose in question is already in the $\mathrm{C} 2$ '-endo conformation (Fig. 1), preferred by the deoxy-modification. On the other hand, it abolishes the $2^{\prime} \mathrm{OH}$ to phosphate hydrogen bonding and also interferes with the $\mathrm{K}^{+}$binding in the quadruplex groove (the O2' atom is involved in this interaction; Fig. 1). Thus, it may affect the stability of the "reversed" U-tetrad motif.
The other introduced sugar modification (M2) involves the introduction $2^{\prime} \mathrm{OMe}-\mathrm{U}$ residue. While still negating the $2^{\prime} \mathrm{OH}$ to phosphate hydrogen bonding, this modification enforces the $\mathrm{C}^{\prime}$-endo conformation and possibly causes some steric hindrance. It may thus induce more pronounced structural changes within the 3 '-terminal U-tetrad.

Certainly, hydrogen bonding between the uridine residues within the $U$-tetrad is also among the most important factors keeping the 3 '-terminus of the quadruplex wellstructured. In order to quantify its contribution, we prepared a sequence mutant bearing a cytidine at the $3^{\prime}$-terminus (M3). The substitution of the $\mathrm{O} 4$ atom of the uridine-the acceptor of the hydrogen bond between the uridines-for an $\mathrm{NH}_{2}$ group should selectively abolish the hydrogen bonding within the tetrad.

To investigate whether the steric repulsion within T-tetrad contributes to its reduced stability, we introduced the 5-methyl-ribouridine (5MeU) modification (M4). Such a modification should not influence any of the other monitored interactions. Together with $\mathrm{M} 1$ it should also allow to verify which of the two differences between DNA and RNA, the presence of the methyl group or the lack of the $2^{\prime} \mathrm{OH}$ hydroxyl group, is mostly responsible for the lower stability of the $3^{\prime}$-terminal T-tetrads in DNA. To quantify the total effect of the two modifications in the current structural context we also synthesized a variant containing a deoxythymidine residue at the 3 '-terminus (M5), or-in other words - the sum of the modifications M1 and M4. Finally, using a LNA-thymidine $\left({ }^{\mathrm{LNA}} \mathrm{T}\right)$ modification $(\mathrm{M} 6)$, we verified whether the introduction of an LNA modification can at least partially stabilize the 3 '-terminal T-tetrad. The LNA modification is usually associated with a pronounced stabilizing effect when introduced into helical context. However, it also locks the modified residue in the C3'endo conformation and at the same time abolishes the possibility of 2' $\mathrm{OH}$-to-phosphate hydrogen bonding within the "reversed" U-tetrad (Fig. 1). The LNA sugar also features an additional $-\mathrm{O}-\mathrm{CH}_{2}$ - linker group which may increase the steric demands of the modified residue but in the "reversed" conformation this group should be directed outwards from the molecule (see Supplemental Fig. S1).

All the modifications discussed above were aimed at selectively destabilizing interactions important for the stability of the "reversed" U-tetrad motif. Conversely, in an attempt to additionally stabilize this motif, we designed a sequence bearing a pseudouridine residue at its $3^{\prime}$ terminus (M7). In pseudouridine, the N1 atom (with its attached $\mathrm{H} 1$ hydrogen) takes the spatial position occupied by the C5 atom in a standard uridine residue. We expected that this modification might allow for the formation of an additional hydrogen bond per pseudouridine residue (N1-H1...O4); see Supplemental Fig. S1), leading to a much more stable tetrad. On the other hand, the original "reversed" U-tetrad conformation features a C2'-endo sugar conformation which may be important for its 


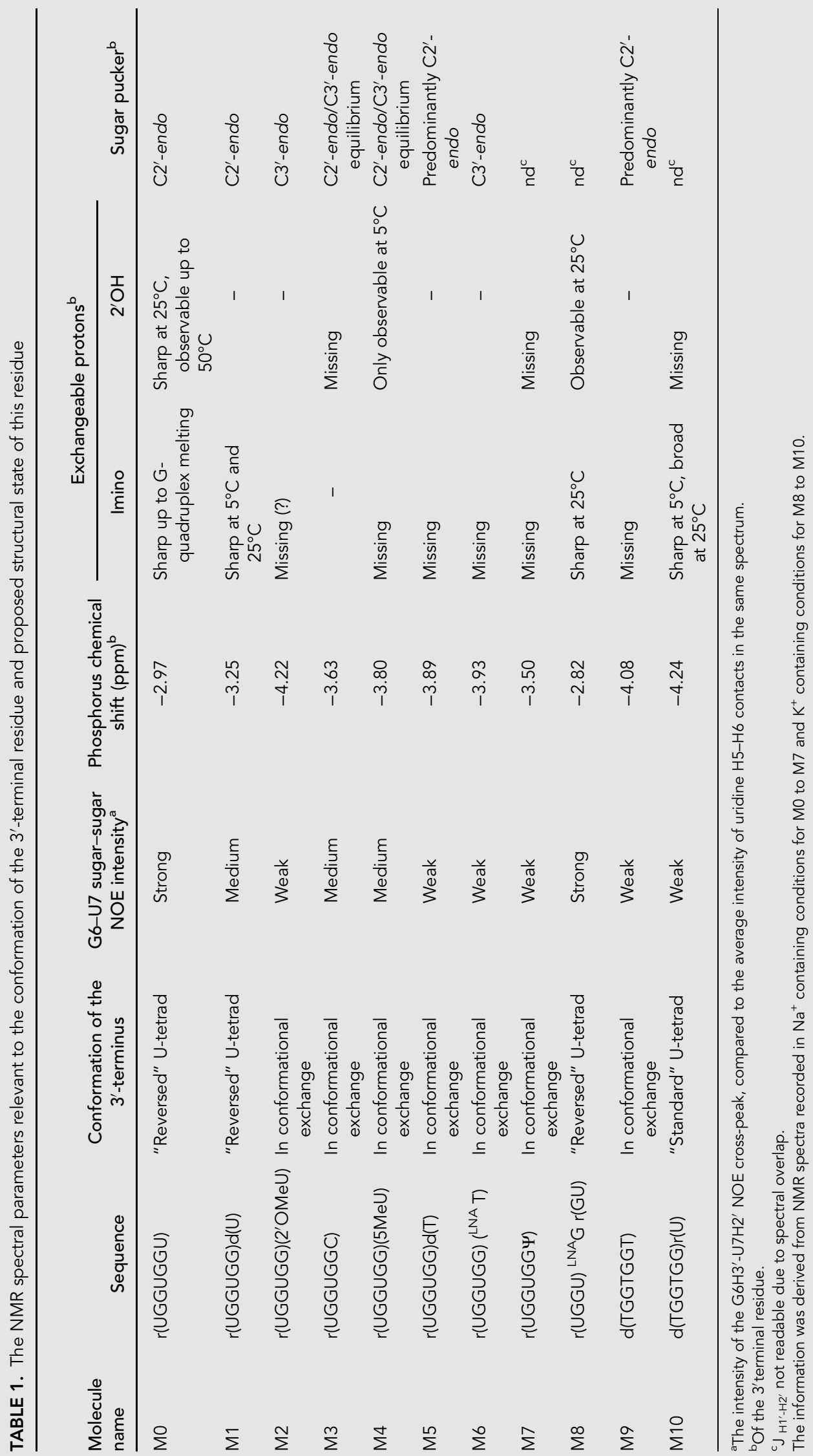


stabilizing effect, while a pseudouridine residue is known to prefer a C3'-endo sugar pucker (Davis 1995).

We also synthesized an additional oligonucleotide containing an LNA modification at the G5 position (M8). The results obtained for this molecule turned out to provide an interesting point of reference to interpret the observations we made for the quadruplexes modified at the $3^{\prime}$ terminus.

We were also interested whether the formation of the "reversed" U-tetrad requires the presence of an RNA quadruplex scaffold or whether it can also occur when a 3 -terminal uridine residue is introduced into a DNA quadruplex as it may provide a way to stabilize tetramolecular DNA G-quadruplexes as well. To answer this question, a DNA analog, bearing a single RNA residue at the $3^{\prime}$-termi-
nus_-d(TGGTGG)r(U)—was synthesized (M10). As a reference point for the melting and NMR analyses, the full DNA quadruplex d(TGGTGGT) was also investigated (M9).

\section{UV melting experiments}

The apparent melting temperatures $\left(T_{m}{ }^{*}\right)$ measured for each G-quadruplex in sodium and potassium containing buffers are listed in Table 2, while the melting curves are presented in Supplemental Figure S2 and Supplemental Figure S3. It can be seen that all the introduced 3'-terminal modifications (M1-M7) noticeably destabilize the RNA quadruplex fold. These destabilizing modifications can be divided into two tiers. The M1 modified oligo has $\mathrm{Tm}^{*} \sim 10^{\circ} \mathrm{C}$ lower than the original quadruplex, while for the remaining ones (M2-M7) the $\Delta \mathrm{T}_{\mathrm{m}}{ }^{*}$ is closer to $20^{\circ} \mathrm{C}$. It is also interesting to note that the described trends repeat themselves very well under both $\mathrm{Na}^{+}$and $\mathrm{K}^{+}$containing conditions. The internal G5-LNA modification (M8) turned out not to appreciably influence the melting of the quadruplex. As far as the DNA quadruplexes (M9 and M10) are concerned, their melting temperatures turned out to be appreciably lower than those of their RNA counterparts, as may have been expected. The introduction of the 3 '-terminal RNA residue in $\mathrm{M} 10$ resulted in a moderate increase in the melting temperature compared to $\mathrm{M} 9\left(\sim 6^{\circ} \mathrm{C}-7^{\circ} \mathrm{C}\right)$.

\section{NMR structural analysis}

In order to determine the structural rearrangements induced by each modification, all the modified quadruplexes were analyzed by NMR spectroscopy. The NMR analysis was performed in both $\mathrm{Na}^{+}$and $\mathrm{K}^{+}$containing buffers. Just as for the nonmodified quadruplex, the chemical shifts of nonexchangeable protons were very similar between the two conditions for all the $3^{\prime}$-modified RNA quadruplexes (M1-M7). However, in the current study the samples dissolved in the sodium-containing buffer yielded in general cleaner spectra and therefore these conditions were selected for further analysis. Portions of 1D ${ }^{1} \mathrm{H}-\mathrm{NMR}$ spectra recorded for the G-quadruplexes 
TABLE 2. The results of UV-melting experiments for quadruplexes MO-M10

\begin{tabular}{|c|c|c|c|c|c|c|}
\hline \multirow[b]{2}{*}{ Sample } & \multicolumn{3}{|c|}{$150 \mathrm{mM} \mathrm{NaCl}$} & \multicolumn{3}{|c|}{$50 \mathrm{mM} \mathrm{KCl}$} \\
\hline & $\mathrm{T}_{\mathrm{m}}{ }^{*} 260 \mathrm{~nm}$ & $\mathrm{~T}_{\mathrm{m}}{ }^{*} 295 \mathrm{~nm}$ & $\Delta \mathrm{T}_{\mathrm{m}}{ }^{*} 260 \mathrm{~nm}^{\mathrm{c}}$ & $\mathrm{T}_{\mathrm{m}}{ }^{*} 260 \mathrm{~nm}$ & $\mathrm{~T}_{\mathrm{m}}{ }^{*} 295 \mathrm{~nm}$ & $\Delta T_{m}^{*} 260 n^{c}$ \\
\hline MO & 61.8 & 61.9 & - & 87.0 & 86.9 & - \\
\hline M1 & 51.9 & 51.9 & -9.9 & 77.8 & 77.9 & -9.2 \\
\hline M2 & 44.1 & 44.6 & -17.7 & 74.1 & 73.8 & -12.9 \\
\hline M3 & 39.9 & 39.9 & -21.9 & $-^{\mathrm{a}}$ & $-^{a}$ & $-^{a}$ \\
\hline M4 & 43.1 & 42.9 & -18.7 & 69.9 & 70.6 & -17.1 \\
\hline M5 & 43.4 & 43.7 & -18.4 & $\sim 70$ & $\sim 70$ & $\sim-17$ \\
\hline M6 & 43.4 & 42.8 & -18.4 & 69.8 & 70.6 & -17.2 \\
\hline M7 & 44.2 & 44.8 & -17.6 & 73.2 & 74.2 & -13.8 \\
\hline M8 & 60.5 & 61.3 & -1.3 & $>90.0$ & $>90.0$ & $>3.0$ \\
\hline M9 & $-{ }^{\mathrm{b}}$ & $-{ }^{\mathrm{b}}$ & $-{ }^{\mathrm{b}}$ & 55.8 & 55.8 & - \\
\hline M10 & $-{ }^{\mathrm{b}}$ & $-{ }^{b}$ & $-{ }^{b}$ & 62.0 & 62.2 & 6.2 \\
\hline
\end{tabular}

The oligomer M3 formed a complex mixture of G-quadruplex folded states in the presence of $\mathrm{K}^{+}$, as evidenced by the NMR spectra.

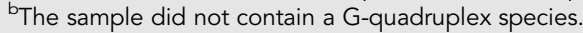

With respect to M0 for RNA G-quadruplexes and M9 for DNA G-quadruplexes.

The apparent melting temperatures $T_{m}{ }^{*}\left({ }^{\circ} \mathrm{C}\right)$ were independently derived from the melting curves measured at 260 and $295 \mathrm{~nm}$.

$\mathrm{MO}-\mathrm{M} 7$ in $\mathrm{Na}^{+}$containing conditions are presented in Figure 3, while the corresponding NOESY data is gathered in Supplemental Figure S4.

The recorded spectra allowed us to establish that the $3^{\prime}$ terminal modifications (M1-M7) did not induce any global structural transitions. All the meaningful chemical shift changes $(\triangle C S$ ) with respect to the nonmodified quadruplex (M0) were strictly localized at the 3 'terminal $\mathrm{G} 6$ and $\mathrm{U} 7$ residues (Fig. 3; Supplemental Tables S1, S2). This indicates that the $5^{\prime}$-terminal and central parts of the quadruplexes remained unaffected by the introduced modifications. On the other hand, the structures of the $3^{\prime}$-terminal region varied greatly between G-quadruplexes. The following spectral features were used to establish the presence and rigidity of the "reversed" U-tetrad motif in a given quadruplex:

1. Chain reversal brings the sugars of G6 and U7 close to each other, giving rise to several prominent intersugar NOEs. The U7-H2' to G6-H3' contact is the strongest among them: its volume for the nonmodified quadruplex is comparable to the $\mathrm{H} 5-\mathrm{H} 6$ cross-peaks of uridines (2.5 ^ distance). The disappearance of this intersugar NOE can be used as a clear indication that chain reversal does not take place.
2. The unusual values of $\alpha$ and $\zeta$ dihedral angles present in the "reversed" U-tetrad conformation are reflected in the chemical shift of the phosphorus atom involved. When chain reversal takes place the phosphorus atom of U7 resonates at $\sim-2.95$ ppm, over 1 ppm away from the range of shifts typical of the regular A-type conformation ( -4 to $-5 \mathrm{ppm}$ ) (Gorenstein 1984). The

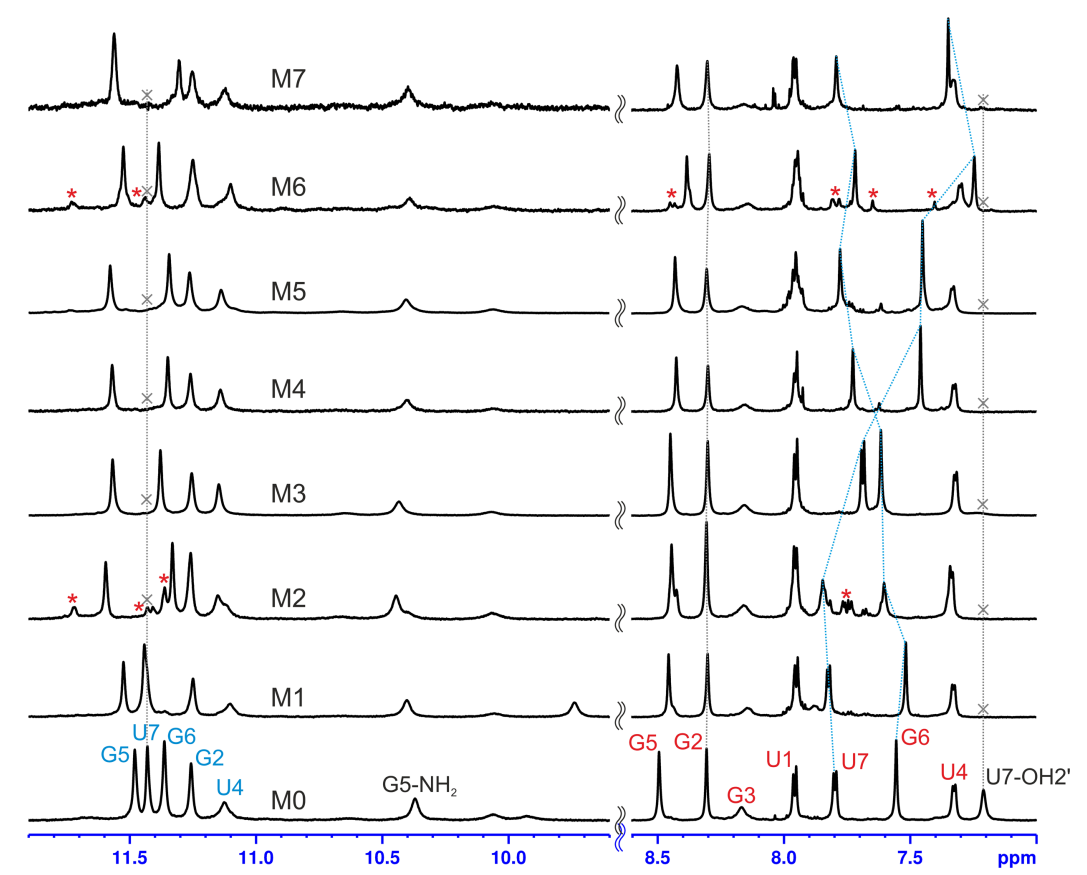

FIGURE 3. The ${ }^{1} \mathrm{H}-\mathrm{NMR}$ spectra (imino and aromatic regions) of G-quadruplexes MO-M7 recorded in $\mathrm{Na}^{+}$containing conditions at $25^{\circ} \mathrm{C}$. The blue dotted lines follow some among the resonances with the greatest chemical shift changes. Additional NMR signals from minor species present in some of the samples are marked with asterisks. 
perturbation of the chain reversal can be expected to shift the U7 phosphorous resonance back toward the typical range.

3. For the "reversed" U-tetrad geometry, the sugar of the 3'-terminal uridine assumes an $\mathrm{C}^{\prime}$ '-endo, as observed in multiple instances in the crystal structures. In $r(U G G U G G U)_{4}(\mathrm{MO})$ the $\mathrm{U} 7-\mathrm{H}^{\prime}{ }^{\prime}$ resonance is observed in NMR spectra as a doublet with ${ }^{3} \mathrm{~J}_{\mathrm{H} 1^{\prime}-\mathrm{H} 2^{\prime}} \approx 8 \mathrm{~Hz}$. The reduction of this coupling constant would indicate the transition of the U7 sugar from $\mathrm{C}^{\prime}$-endo, to a conformational equilibrium (likely $\mathrm{C} 2$ '-endo/C3'-endo), and thus an increased lability of the $3^{\prime}$-terminus of the quadruplex. In the current work the ${ }^{3} \mathrm{~J}_{\mathrm{H} 1^{\prime}-\mathrm{H} 2^{\prime}}$ coupling constants for the modified G-quadruplexes were measured directly from $\mathrm{U} 7-\mathrm{H}^{\prime}{ }^{\prime}$ resonances, in most cases well-resolved in the 1D NMR spectra (presented in Supplemental Fig. S5).

4. In the nonmodified structure, both the $\mathrm{H} 3$ and $2^{\prime} \mathrm{OH}$ protons of U7 are very inert thanks to stable hydrogen bonding interactions. The disappearance of either of these protons (due to solvent exchange) would indicate an increased lability, occurring in the base (imino) and/ or sugar $\left(2^{\prime} \mathrm{OH}\right)$ regions of the "reversed" U-tetrad. In our previous work we postulated that the acceptor of the hydrogen bond from $\mathrm{U} 7-\mathrm{OH} 2^{\prime}$ is the $\mathrm{G} 6$ phosphate group (Andrałojć et al. 2019). Since then, we performed additional NMR measurements, as proposed by the Wöhnert group (Duchardt-Ferner et al. 2011; Duchardt-Ferner and Wöhnert 2017), to directly identify the hydrogen bond acceptor in the original G-quadruplex M0. The through hydrogen bond ${ }^{1} \mathrm{H}-{ }^{31} \mathrm{P}$ correlation spectrum presented in Supplemental Figure S6, unequivocally confirms the G6 phosphate group as the acceptor of hydrogen bond from $\mathrm{U} 7-\mathrm{OH} 2^{\prime}$.

The most relevant NMR parameters measured for each modified G-quadruplex are gathered in Table 1 and described in detail below:

\section{M1-dU7}

The deoxyuridine modification induced only minimal structural changes with respect to $\mathrm{MO}$, as demonstrated by several independent indicators. First, no significant chemical shift changes occurred for nonexchangeable protons outside of the modified sugar ring (Supplemental Table S1). For the $\mathrm{H}^{\prime}$ ' to $\mathrm{H}^{\prime}$ ' protons of the U7 deoxyribose, the measured $\triangle \mathrm{CS}$ are easily ascribable to their changed chemical environment. Second, the NOE contacts between the G6 and U7 sugars, characteristic for the "reversed" conformation, are clearly observable. However, the sugar-sugar distance appears to be slightly higher in the modified quadruplex, as the intensity of the U7-H2' to G6-H3' contact is somewhat reduced (to approximately $2 / 3$ of the $\mathrm{H} 5-\mathrm{H} 6$ cross-peaks). This change might be related to the loss of the anchoring effect of the $2^{\prime} \mathrm{OH}$ to phosphate hydrogen bonding, abolished by the 2 '-deoxy- substitution. Third, the chemical shift of the U7 phosphorus atom is only moderately perturbed with respect to the original structure ( 0.28 ppm upfield shift). Lastly, the sugar moiety remained locked in the C2'endo conformation (both the ${ }^{3} \mathrm{~J}_{\mathrm{H} 1^{\prime}-\mathrm{H} 2^{\prime}}$ and the ${ }^{3} \mathrm{~J}_{\mathrm{H} 1^{\prime}-\mathrm{H} 2^{\prime \prime}}$ $\sim 7.5-8 \mathrm{~Hz}$ ) and the imino proton of $\mathrm{H} 3$ gave rise to a sharp resonance both at $5^{\circ} \mathrm{C}$ and $25^{\circ} \mathrm{C}$. These observations confirm that the "reversed" U-tetrad is formed in the M1 quadruplex and remains relatively rigid.

\section{M2-2'OMe-U7}

On the other hand, the M2 modification appears to noticeably reshape the $3^{\prime}$-terminal part of the quadruplex. The $\Delta C S$ with respect to the original quadruplex are not very pronounced, yet they are clearly present also outside the $2^{\prime} \mathrm{OMe}-\mathrm{U} 7$ ribose ring (for example, for $\mathrm{G} 6 \mathrm{H}^{\prime}$ ). The imino proton of U7 is not observed even at $5^{\circ} \mathrm{C}$, indicating an increased lability of uridine bases or even no tetrad formation at all. Most importantly, the NOE contacts between the $\mathrm{G} 6$ and $U 7$ riboses are severely reduced, with the $\mathrm{U} 7-\mathrm{H}^{\prime}$ ' to $\mathrm{G} 6-\mathrm{H} 3^{\prime}$ cross-peak almost not observable. The perturbation of the $3^{\prime}$-terminal part of the molecule is also reflected in the phosphorus chemical shifts. The phosphorus atom of U7 is shifted upfield by $\sim 1.25$ ppm with respect to M0. Also, the previous two phosphate groups, G6 and G5, are affected by $\sim 0.5$ ppm, see Supplemental Table S2. Overall, the 3 '-terminal uridine in M2 is conformationally mobile, possibly quickly alternating between both unpaired and tetrad-forming states.

Moreover, the NMR spectra revealed the presence of one or more minor species, in slow exchange, with a total population of 20\%-30\% (Fig. 3). These species shared the same overall quadruplex fold with the major form, yet differed in the conformation of their 3 '-terminal parts (the resonances belonging to U1 up to U4/G5 were completely superimposed for all the forms present, while those of G6 and U7 displayed a marked variability in their chemical shifts). Due to their weak NMR signals, it was not possible to gain any insights into the structural details of these minor conformations.

\section{M3-rC7}

The substitution of the $U 7$ residue into cytidine abolishes the hydrogen bonding in the $3^{\prime}$-terminal tetrad and causes the $3^{\prime}$-terminal residue to become conformationally flexible. Interestingly however, the conformational equilibrium of the $3^{\prime}$-terminal $C$ residue still appears to involve a significant population of states featuring chain reversal. These assertions are made based on the following NMR observations. The presence of a significant population of the inverted conformation(s) was evident from the characteristic, strong NOE contacts between sugar moieties of 
G6 and C7, with intensities just slightly weaker than those observed for M1. However, the other NMR parameters indicated that $\mathrm{C} 7$ is certainly not rigidly fixed in the "reversed" conformation. The U7-2'OH proton was not observable even at $5^{\circ} \mathrm{C}$, while the ribose moiety of $\mathrm{C} 7$ in the M3 quadruplex was clearly undergoing fast conformational exchange, as evident from the reduced ${ }^{3} \mathrm{~J}_{\mathrm{H} 1^{\prime}-\mathrm{H} 2^{\prime}}$ of $\sim 5.5 \mathrm{~Hz}$. Moreover, no signs of hydrogen bonding between the cytidine bases (to form a C-tetrad) were observed. The phosphorus resonance of $\mathrm{C} 7$ appears at -3.63 ppm, approximately halfway between the chemical shift value observed for $\mathrm{MO}$ and the one expected for a standard helical conformation. Overall, a plausible interpretation of the spectral data for M4 involves a conformational equilibrium between conformation(s) with chain reversal and other states probably featuring unpaired cytidines.

\section{M4-5Me-U7}

The introduction of the 5Me group into U7 (M4) is a minor modification, leading only to some steric crowding, without directly interfering with any of the tetrad stabilizing interactions. Interestingly though, the structural consequences of its presence appear to be quite similar to those of the cytidine substitution (M3) - an increase of the lability of the 3 '-terminal $5 \mathrm{Me}$-U-tetrad, yet without completely abolishing the chain reversal. The presence of a population of the inverted conformation was evident from the characteristic, strong NOE contacts between G6 and U7 (with intensities comparable to those we observed for M1). The increased lability, on the other hand, manifested itself in a multitude of features. The imino proton of $5 \mathrm{Me}-\mathrm{U} 7$ was absent in the NMR spectra even at $5^{\circ} \mathrm{C}$ (Fig. 3). The protection of $\mathrm{U} 7-2^{\prime} \mathrm{OH}$ proton from exchange with the solvent was also greatly reduced, as its resonance was only observable at a low temperature $\left(5^{\circ} \mathrm{C}\right)$. Moreover, the ribose of $5 \mathrm{Me}-\mathrm{U} 7$ was clearly undergoing fast conformational exchange, as evident from the reduced ${ }^{3} \mathrm{~J}_{\mathrm{H} 1^{\prime}-\mathrm{H} 2^{\prime}}$ of $\sim 5 \mathrm{~Hz}$. These last two observations indicate that the stabilizing $\mathrm{U} 7-2^{\prime} \mathrm{OH}$ to phosphate hydrogen bonding occurs at best only transiently. The ${ }^{31} \mathrm{P}-\mathrm{NMR}$ chemical shift of $5 \mathrm{Me}-\mathrm{U} 7$ phosphorus is $-3.8 \mathrm{ppm}$, already quite close to the standard -4 to $-5 \mathrm{ppm}$ range. This corresponds to a 0.83 ppm upfield shift with respect to the nonmodified G-quadruplex featuring the "reversed" U-tetrad. Taken together, the NMR data may be explained by an equilibrium between a population of conformers featuring chain reversal (and perhaps a well-formed "reversed" U-tetrad) and other states involving either unpaired uridines or the presence of a "standard" U-tetrad.

\section{M5-dT7}

The substitution of U7 for a deoxythymidine corresponds to the combined introduction of modifications M1 and
M4. As M1 was shown to have no significant structural effect, it is not surprising that M5 turned out to behave very similarly to M4, yet with a somewhat reduced fraction of "reversed" states present. Just like for M4 the dT7 imino proton was not observable even at $5^{\circ} \mathrm{C}$. Also, the phosphorus chemical shift of $-3.89 \mathrm{ppm}$ is very similar to that measured for M4 (a further shift of $~ 0.1 \mathrm{ppm}$ toward the standard region). Once again, NOE contacts between the G6 and dT7 sugar moieties were observed, reporting on at least some population of inverted conformations. However, these NOEs are now significantly weaker. This NOE intensity reduction could indicate a lower population of states with chain reversal. One has to, however, be very cautious when dealing with NOE intensities measured for parts of the molecule undergoing conformational variability, as without knowing the details of the motions involved it is not really possible to interpret them in a (semi)quantitative manner. Thus, the conclusion about the reduced population of "reversed" states should be treated as just one possibility of explaining the data, corroborated by a further slight shift of the dT7 phosphorus toward the standard chemical shift range. Overall, one can conclude that the $3^{\prime}$-terminal residue of M5 is probably quite labile assuming both conformations involving a chain reversal and ones with more standard dihedral angle values.

\section{M6- ${ }^{\text {LNA } T 7}$}

The ${ }^{\text {LNAT7 }}$ modified G-quadruplex appears to once again feature a predominantly conformationally labile 3 '-terminus, with a small fraction of "reversed" conformations present. This conclusion can be made based on the fact that the imino proton of the ${ }^{\mathrm{LNA}} \mathrm{T7}$ residue cannot be observed even at $5^{\circ} \mathrm{C}$, while the ${ }^{31} \mathrm{P}$ chemical shift of this residue is almost identical to what was observed for M5 (-3.93 ppm). Also the characteristic NOEs between the sugar moieties of the two terminal residues were observed with reduced intensities, similar to those measured for M5. The sugar conformation in an LNA residue is fixed to $\mathrm{C} 3^{\prime}$-endo and the $2^{\prime} \mathrm{OH}$ group is missing, thus these features cannot be used to assess the conformational state of the residue. Overall, the gathered NMR data point toward a labile $3^{\prime}$-terminal residue, behaving very similarly to that of M5.

Apart from the main form, the NMR sample of M6 contained an additional oligonucleotide species, with a population of $\sim 10 \%-15 \%$. In contrast to the minor form encountered previously for $\mathrm{M} 2$, the current one probably features a completely different fold of the entire chain as its chemical shifts appear to differ from those of the main conformer all throughout the sequence. The low population of this species precluded a structural analysis. 


\section{M7- $\boldsymbol{\Psi} 7$}

The $\Psi$ residue was introduced at the $3^{\prime}$-terminus of the Gquadruplex in the hope that it would form an even more stable tetrad, held together by two hydrogen bonds per residue. However, the NMR analysis revealed that the $\Psi 7$ modification actually gives rise to a partially disordered 3'-terminal region, likely similar to M5 and M6. Both the $\Psi 7$ imino and $2^{\prime} \mathrm{OH}$ proton resonances are not observable even at $5^{\circ} \mathrm{C}$, while the $\Psi 7$ phosphorus resonates at -3.50 ppm. The NOEs between the sugar moieties of $\mathrm{G} 6$ and $\Psi 7$ are observable, but their intensities are quite weak. All together these data suggest the lack of stable $\Psi$-tetrad formation.

\section{M8- ${ }^{\text {LNA }}$ G5}

The introduction of LNA in position G5 has a significant effect on the proton chemical shifts in the central part of the quadruplex, especially those of U4. Both $\mathrm{H} 5$ and $\mathrm{H} 6$ aromatic protons of this residue are downfield shifted by 1.41 and 0.77 ppm, respectively (Supplemental Fig. S7). Such a strong $\triangle \mathrm{CS}$ is a clear indication of the loss of shielding, provided by the ring currents in the neighboring guanine bases (Patel and Hosur 1999). This in turn suggests that the $U 4$ residues are bulged out from the quadruplex stem in M7. This hypothesis is strongly supported by the disappearance of the U4 imino proton from the NMR spectra, as well as by the presence of a series of new NOE contacts between the $\mathrm{G} 3$ and $\mathrm{G} 5$ residues (Supplemental Fig. S8). On the other hand, the conformation of the $5^{\prime}$ - and $3^{\prime}$-terminal parts of the quadruplex ap-

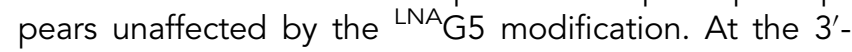
terminus all the important G6-U7 contacts are observable at full strength. Both the $\mathrm{U} 7-\mathrm{H} 3$ and $\mathrm{U} 7-2^{\prime} \mathrm{OH}$ protons give rise to sharp resonances, the $\mathrm{U} 7$ ribose adopts a pure $\mathrm{C}^{\prime}$ endo conformation and the phosphorus chemical shift of U7 (-2.82 ppm) indicates chain reversal. Thus, overall, in M7 the "reversed" U-tetrad is present in its most generic form.

\section{M9-d(TGGTGGT)}

In contrast to the above described RNA G-quadruplexes, the all-DNA construct, M9, turned out to behave very differently in $\mathrm{Na}^{+}$and $\mathrm{K}^{+}$containing buffers. In the $\mathrm{Na}^{+}$containing conditions no G-quadruplex formation was observed at all. No imino proton resonances were observable, while the aromatic and anomeric protons displayed a very low resonance frequency dispersion (Supplemental Fig. S9), characteristic to disordered random-coil-like conformational states. This picture did not change even after several months of incubation in the NMR buffer, urging us to conclude that M9 has no propensity for G-quadruplex formation in the presence of $\mathrm{Na}^{+}$. In contrast, when $\mathrm{K}^{+}$is present in the buffer, a tetramolecular parallel G- quadruplex is formed (Supplemental Fig. S9). Four sharp imino protons are observable in the 10-12 ppm range, indicating G-quadruplex formation and only a single set of aromatic protons is present, indicating that the G-quadruplex has a fourfold symmetry achievable only by the parallel tetramolecular fold (Cheong and Moore 1992). Following similar procedures as for the RNA quadruplexes, the imino resonances can be identified to belong to $G 2$, G3, G5, and G6. In addition, a broader resonance at 9.5 ppm can be assigned to the T4 imino proton, indicating that the central thymidine is involved in tetrad formation, as was already observed for several similar DNA sequences. However, there is no indication that either T1 or T7 terminal thymidine residues may form T-tetrads, as their imino protons cannot be observed even at $5^{\circ} \mathrm{C}$. This is consistent with the literature data, described in the Introduction, that terminal T-tetrads generally do not form or remain highly labile.

\section{M10-d(TGGTGG)r(U)}

The M10 DNA oligonucleotide modified with a ribouridine residue at the $3^{\prime}$-terminus behaves similarly to its all-DNA counterpart $\mathrm{M} 9$ in the $\mathrm{Na}^{+}$containing conditions. The lack of observable imino protons and low resonance dispersion of other protons strongly point toward a disordered, random-coil-like conformation in these conditions (Supplemental Fig. S9). In turn, in the $\mathrm{K}^{+}$buffer, the presence of a well-formed parallel tetramolecular G-quadruplex was observed just like for M9 (Supplemental Fig. S9). Resonance assignment and NOE analysis reveal that the core of the quadruplex is the same as for M9. However, this time the formation of an additional tetrad is ascertained by the presence of one more imino resonance (Supplemental Fig. S9). This resonance can be assigned to U7, based on its strong NOE contact to U7H5. The $\mathrm{U} 7 \mathrm{H} 3$ resonance is very sharp at $5^{\circ} \mathrm{C}$, but broadens significantly with increasing temperature and is already barely observable at $\sim 30^{\circ} \mathrm{C}$. This means that the $3^{\prime}$-terminal U7 tetrad is rather thermally unstable when present in a DNA G-quadruplex, in sharp contrast with its exquisite stability in RNA G-quadruplexes. A closer inspection of the NMR data for M10 reveals that the $3^{\prime}$-terminal U-tetrad actually adopts very different conformations when present in DNA and RNA. First, the chemical shift of U7 phosphorus in $\mathrm{M} 10$ is -4.24 ppm, well within the standard range for helical conformations, suggesting that no chain reversal takes place. Second, only very weak NOE contacts are observed between the $\mathrm{U} 7 \mathrm{H} 2^{\prime}-\mathrm{G} 6 \mathrm{H}^{\prime}$, once again suggesting that a conformation with chain reversal cannot be present in the system for a significant fraction of time. Third, the $\mathrm{U} 7 \mathrm{OH} 2^{\prime}$ resonance is not observable even at $5^{\circ} \mathrm{C}$, suggesting that in $\mathrm{M} 10$ this proton is not involved in any hydrogen bonding interaction. Overall, the NMR data strongly suggest that while a U7 tetrad does form in the DNA G- 

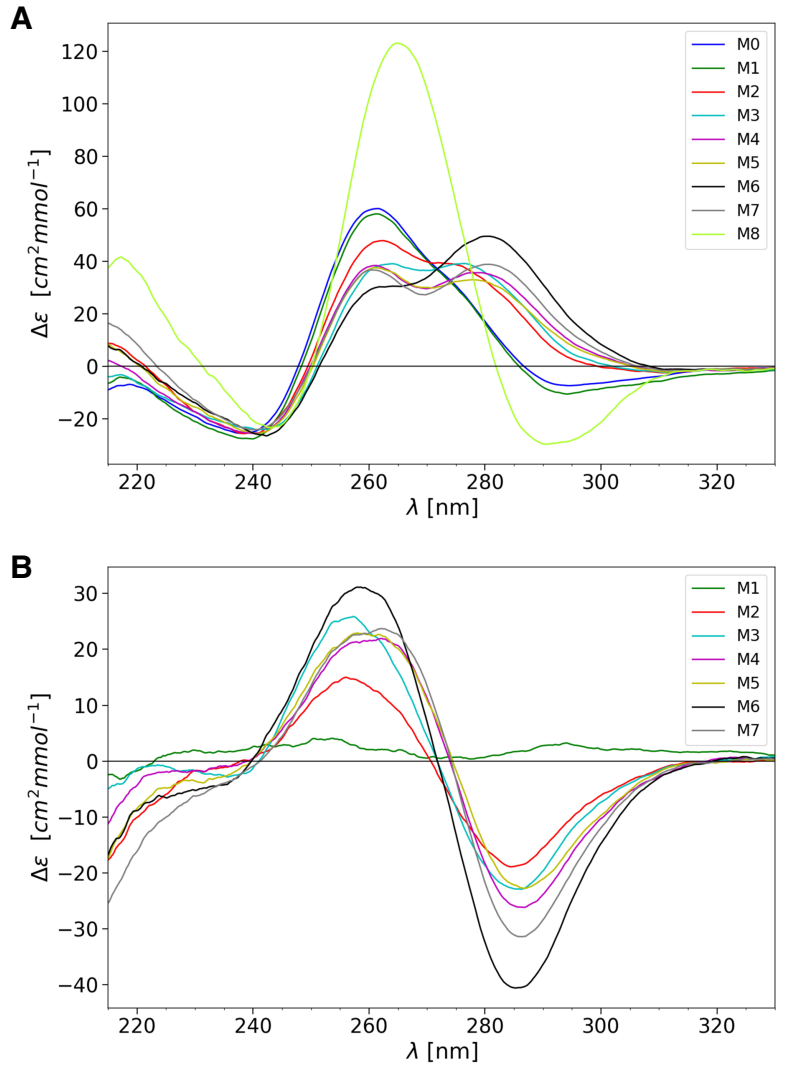

FIGURE 4. (A) The CD spectra recorded for G-quadruplexes MO-M8 in $\mathrm{Na}^{+}$containing conditions at $25^{\circ} \mathrm{C} ;(B)$ the difference between the CD spectrum of each $3^{\prime}$-modified G-quadruplex (M1-M7) and that of the reference one $(\mathrm{MO})$.

quadruplex it does so without involving a reversal of the oligonucleotide chain and that this structural element is not particularly stable.

\section{CD spectroscopy}

As a secondary method of assessing the structural changes induced by the various modifications we collected the CD spectra for all the studied quadruplexes, M0-M10, in both $\mathrm{Na}^{+}$and $\mathrm{K}^{+}$containing buffers. The samples used for $\mathrm{CD}$ spectroscopy were obtained directly from the material previously used for the NMR studies, by a simple dilution. All the CD spectra collected in $\mathrm{Na}^{+}$and $\mathrm{K}^{+}$containing conditions are gathered in Figure 4A and Supplemental Figure $\mathrm{S} 10 \mathrm{a}$, respectively. Direct comparisons between the $\mathrm{Na}^{+}$ and $\mathrm{K}^{+}$spectra for the same construct can be found in Supplemental Figure S11. For a set of molecules, differing only by subtle site specific modifications, the collected CD spectra display quite a surprising degree of diversity. While all the spectra share an ellipticity maximum $\sim 260$ $\mathrm{nm}$, typical of parallel-stranded G-quadruplexes (del Villar-Guerra et al. 2018), the spectral behavior at higher wavelengths is more surprising. One subset of the studied
G-quadruplexes, namely $M 0, M 1$, and $M 8$, give rise to $C D$ spectra with a minimum $\sim 290 \mathrm{~nm}$, while the remaining molecules display a second maximum $\sim 280 \mathrm{~nm}$ in their CD spectra, instead. None of these two behaviors is what one would expect from a typical parallel-stranded G-quadruplex which, in general, does not display any significant ellipticity above $280 \mathrm{~nm}$. The shape of the spectra displaying two maxima is actually very reminiscent of what one would expect for a hybrid-type G-quadruplex fold, instead (del Villar-Guerra et al. 2018). As a separate note, it is worth bringing forward that for any given studied molecule, the CD spectra recorded in $\mathrm{Na}^{+}$and $\mathrm{K}^{+}$conditions are similar (Supplemental Fig. S11), yet with a clear trend that the maximum $\sim 280 \mathrm{~nm}$ is always stronger in the presence of $\mathrm{Na}^{+}$at the expense of the other maximum.

\section{MD simulations}

In order to verify from another angle which energetic contributions favor the "reversed" U-tetrad conformation over a typical one, we performed molecular dynamics (MD) simulations on the r(UGGUGGU) ${ }_{4}$, followed by an MM-PBSA analysis. The dynamics of $\mathrm{MO}$ was simulated starting from two very distinct initial conformations: (A) its native state with a "reversed" U-tetrad (taken from PDBID: 6GE1) and $(B)$ an alternative state in which the $U 7$ residue forms a typical U-tetrad (see Materials and Methods). The subsequent MM-PBSA analysis of both trajectories revealed that system A with "reversed" U-tetrad has indeed a lower free energy compared to $B$ (by $13.9 \mathrm{kcal} / \mathrm{mol}$ ), in accordance with the experimental observation. To better understand the source of this energy difference, the total energies of each system were decomposed into contributions from the various components of the MM force field (bond, angle and dihedral strain, van der Waals interactions, intramolecular electrostatic energies) and solvation energy (electrostatic contribution and a surface-area-dependent term for nonelectrostatic contributions to solvation), as shown in Table 3. While the bond length and angle deformation energies turned out to be very comparable for the two systems, the dihedral term is somewhat disfavoring the "reversed" U-tetrad. This might have been expected knowing that this conformation contains several atypical torsion angle values. Similarly, the solvation energy is significantly better for the typical U-tetrad. On the other hand, however, the intramolecular van der Waals and especially electrostatic interactions strongly favor the "reversed" U-tetrad conformation. When exact numbers are compared these last terms are enough to outweigh the unfavorable solvation and dihedral contributions and make the "reversed" U-tetrad the preferred conformation. The less computationally demanding variant of the analysisMM-GBSA — was also performed and brought about virtually the same results (Supplemental Table S3). 
TABLE 3. The results of the MM-PBSA analysis of the MD trajectories recorded for MO in two distinct conformations-with "reversed" and "standard" U-tetrads at the 3'-terminus

\begin{tabular}{|c|c|c|c|c|c|}
\hline Energy component $(\mathrm{kcal} / \mathrm{mol})^{a}$ & "Reversed" U-tetrad (system A) & $\sigma_{M}$ & "Standard" U-tetrad (system B) & $\sigma_{M}$ & Energy difference (A-B) \\
\hline BOND & 240.0 & 0.2 & 240.3 & 0.2 & -0.3 \\
\hline ANGLE & 460.8 & 0.3 & 461.9 & 0.3 & -1.1 \\
\hline DIHED & 692.0 & 0.2 & 684.3 & 0.2 & 7.7 \\
\hline VDW & -572.9 & 0.2 & -561.7 & 0.2 & -11.2 \\
\hline ELE & 1031.4 & 0.6 & 1151.9 & 0.6 & -120.5 \\
\hline 1-4 VDW & 233.2 & 0.1 & 233.8 & 0.1 & -0.6 \\
\hline 1-4 ELE & -2662.2 & 0.3 & -2664.1 & 0.3 & 1.9 \\
\hline EPB & -5104.5 & 0.5 & -5215.6 & 0.5 & 111.1 \\
\hline ENPOLAR & 22.0 & 0.0 & 23.1 & 0.0 & -1.1 \\
\hline G gas & -577.7 & 0.6 & -453.7 & 0.6 & -124.0 \\
\hline G solv & -5082.4 & 0.5 & -5192.5 & 0.5 & 110.1 \\
\hline E polar_total & -6735.3 & & -6727.8 & & -7.5 \\
\hline E np_total & -317.7 & & -304.8 & & -12.9 \\
\hline TOTAL & -5660.1 & 0.4 & -5646.2 & 0.3 & -13.9 \\
\hline
\end{tabular}

aBOND, ANGLE and DIHED denote internal (bond, angle, and dihedral angle, respectively) potential energies. VDW represents nonbonded van der Waals energy, ELE-nonbonded electrostatic energy, 1-4 VDW and 1-4 ELE-energy contribution from the nonbonded interactions (VDW and ELE, respectively) between atoms separated by three consecutive bonds, EPB-electrostatic component to solvation free energy estimated with Poisson-Boltzmann model, ENPOLAR - nonelectrostatic contribution to solvation energy, G gas-total vacuum energy (G gas = BOND + ANGLE + DIHEDRAL + VDW + ELE + 1-4 VDW + 1-4 ELE), G solv—total solvation free energy, (G solv =EPB + ENPOLAR), E polar_total $=E L E+1-4 E L E+E P B_{-}$, E np_total $=V D W+1-4 V D W+E N P O L A R$, $T O T A L=G$ gas $+G$ sol $=$ BOND + ANGLE + DIHEDRAL + E polar_total $+E$ np_total, $\sigma_{M}$-standard error of the mean.

\section{DISCUSSION}

\section{Correlation between conformation of the $3^{\prime}$-terminal residue and RNA G-quadruplex thermal stability}

A combined analysis of NMR spectra and UV melting data allows us to correlate the trends observed in thermal stabilities of the studied G-quadruplexes with changes occurring to their structures. The structural effect of each modification was strictly confined to the 3'-terminal region, in most cases to the modified U7 residue alone. The conformations of the $3^{\prime}$-termini can be divided into two main groups: those featuring only the "reversed" U-tetrad (M0-M1) and those with at least a partially labile 3'-terminal residue (M2-M7). The structural picture from NMR coincides quite well with the stability pattern observed in the UV melting experiments. In the $\mathrm{Na}^{+}$containing buffer, the nonmodified quadruplex, $\mathrm{MO}$, is clearly the most thermally stable $\left(T_{m}=61.8^{\circ} \mathrm{C}\right)$, while the $2^{\prime}$-deoxy modification (M1) — the only one retaining a rigid "reversed" U-tetradinduces the smallest destabilization $\left(\Delta \mathrm{T}_{\mathrm{m}} \approx 10^{\circ} \mathrm{C}\right)$. A majority of the modified $\mathrm{G}$-quadruplexes with labile $3^{\prime}$-termini (M2, M4, M5, M6, and M7) are destabilized by $17^{\circ} \mathrm{C}-$ $18^{\circ} \mathrm{C}$ and only $\mathrm{M} 3$ is destabilized even stronger-by $22^{\circ} \mathrm{C}$. These trends repeat themselves under $\mathrm{K}^{+}$containing conditions (within $\pm 1^{\circ} \mathrm{C}$ of $\Delta \mathrm{T}_{\mathrm{m}}$ ), with only two moderate outliers-M2 and M7-for which the destabilizing effect of the modification is somewhat reduced in the presence of $\mathrm{K}^{+}$(to $13^{\circ} \mathrm{C}-14^{\circ} \mathrm{C}$ ). Thus, regardless of the type of cation present, any modification that interferes with the structural rigidity of the "reversed" U-tetrad motif leads to a decrease of the melting temperature by at least over a dozen degrees (and up to $22^{\circ} \mathrm{C}$ ), emphasizing the important contribution of this element to the G-quadruplex stability. Although we did not attempt to entirely remove the 3'-terminal residue, a literature example of a similar operation performed on the r(UAGGGU) 4 (actually moving the $3^{\prime}$-terminal uridine to the $5^{\prime}$-terminus, where it remains unpaired) reports a $29^{\circ} \mathrm{C}$ reduction of the melting temperature (Xu et al. 2010).

Such a pronounced stabilizing effect is not what one might expect for a structural element kept together by a single hydrogen bond, such as a U-tetrad, and indeed we have postulated before that it is specific only to the U-tetrads present at the 3 '-terminus of the G-quadruplex and adopting the "reversed" U-tetrad conformation. Such a hypothesis appears to be corroborated by the structural and thermodynamic results obtained for Gquadruplex M8 in which the LNA residue was introduced in position G5. This molecule constitutes the only instance in which the central U4 tetrad was selectively abolished. In this case, the removal of the U-tetrad leads to only marginal G-quadruplex destabilization in the presence of $\mathrm{Na}^{+}$ $\left(\sim 2^{\circ} \mathrm{C}\right)$ and even its slight stabilization in $\mathrm{K}^{+}$. The fact that the central U-tetrad can be completely removed without causing a drop in $\mathrm{T}_{\mathrm{m}}$ * suggests that it cannot have a significant positive contribution to G-quadruplex stability. Interestingly, a comparison with some literature results 
suggests that its effect might, in fact, be quite the opposite. The r(UGGGGU) 4 quadruplex was previously reported to melt at above $90^{\circ} \mathrm{C}$, in the presence of $\mathrm{Na}^{+}$(Zhou et al. 2017). A direct comparison of $r(U G G G G U)_{4}$ with our nonmodified $r\left(\right.$ UGGUGGU) 4 quadruplex $\left(\mathrm{MO} ; \mathrm{T}_{\mathrm{m}}{ }^{*}=62^{\circ} \mathrm{C}\right.$ in $\mathrm{Na}^{+}$) suggests that the presence of a central U-tetrad actually appears to have a very pronounced destabilizing effect. The comparison of $\mathrm{M0}$ and M8 G-quadruplexes with r(UGGGGU $)_{4}$ raises an additional question: why M8-with the central uridine residues bulged out and thus sharing the exact same core with r(UGGGGU) 4 (a $5^{\prime}$-U-tetrad, followed by four G-tetrads, followed by a 3'-U-tetrad)—is not similarly stable. Providing a satisfactory answer to this question is, however, out of the scope of the current paper.

Next, we investigated which structural features of the "reversed" U-tetrad contribute the most to its stability and may be considered the driving forces for its formation. The "reversed" U-tetrad conformation involves: (i) a very stable hydrogen bonding between the $2^{\prime} \mathrm{OH}$ group of the $3^{\prime}$-terminal uridine and the phosphate group of the preceding residue; and (ii) an additional binding site for $\mathrm{K}^{+}$(but not $\mathrm{Na}^{+}$) ions in the G-quadruplex groove (Andrałojć et al. 2019). In order to check the role of the $2^{\prime} \mathrm{OH}$ group of the $3^{\prime}$-terminal uridine we designed quadruplex $\mathrm{M} 1$. The lack of a $2^{\prime} \mathrm{OH}$ group does not only eliminate this hydrogen bonding, but also interferes with ion binding. Using NMR spectroscopy, we determined that the "reversed" U-tetrad motif is still preserved in the structure of the M1 quadruplex. Despite this, the thermal stability of the $\mathrm{M} 1$ is lower by $\sim 10^{\circ} \mathrm{C}$ with respect to $\mathrm{M} 0$ under both $\mathrm{K}^{+}$and $\mathrm{Na}^{+}$containing conditions. In the presence of $\mathrm{K}^{+}$, this destabilization can be seen as a combined effect of the missing hydrogen bonding and weakened/missing $\mathrm{K}^{+}$binding in the groove. However, under $\mathrm{Na}^{+}$containing conditions, the destabilizing effect can be fully ascribed to the missing $2^{\prime} \mathrm{OH}$ to phosphate hydrogen bonding, as there is no $\mathrm{Na}^{+}$ion binding even for MO. As the destabilization is similar under the two conditions, it suggests that the contribution of the $\mathrm{K}^{+}$ion binding to the stability of the "reversed" U-tetrad motif is actually negligible. Consequently, it can be concluded that the $2^{\prime} \mathrm{OH}$ to phosphate hydrogen bonding constitutes a major contribution to the stability of the "reversed" U-tetrad. However, it cannot be considered to be the driving force for the formation of the motif itself, as the example of M1 clearly demonstrates that a rigid "reversed" U-tetrad still forms even without this interaction being present.

Another contribution to the stability of G-quadruplexes containing the "reversed" U-tetrad motif is expected to come from the hydrogen bonding within U-tetrad itself. Indeed, this is confirmed by the results we obtained for quadruplex M3 in which the replacement of the 3 '-terminal uridine by cytidine selectively eliminates the possibility of $\mathrm{H} 3 . . . \mathrm{O} 4$ hydrogen bonding between the nucleobases. This replacement does not only lead to the most significant destabilization $\left(22^{\circ} \mathrm{C}\right)$, but also to the loss of the structural integrity of the $3^{\prime}$-terminal tetrad. When the $3^{\prime}$-terminal residue becomes labile, the $2^{\prime} \mathrm{OH}$ to phosphate hydrogen bonding and base stacking with the preceding G-tetrad are also abolished, which is probably partly responsible for the observed destabilization.

The observations presented so far give rise to a major question: Why is the "reversed" tetrad still preferred over a "standard" one, even when additional interactions stabilizing it are abolished (as found for M1)? Among the other structural features that significantly differ between the "reversed" and "standard" U-tetrads one can list: (i) the sugar-phosphate backbone conformation at the G6/ U7 dinucleotide step; (ii) the stacking geometries of the uridine bases with the preceding guanosines; and (iii) hydration patterns around the 3 '-terminal region.

The energetic contribution of the RNA backbone conformation is very unlikely to be in favor of the "reversed" U-tetrad, as it features multiple rotamers rarely observed in RNA. This is confirmed by the MD simulations which revealed noticeably higher energy stored in the dihedral term for the "reversed" U-tetrad (by $8 \mathrm{kcal} / \mathrm{mol}$, Table 3). Thus, the base stacking and hydration contributions remain as the most likely features favoring the "reversed" U-tetrad in M1.

The results of the MD simulations suggest that favorable stacking is a much more likely explanation. The PBSA analysis revealed that a system with a "reversed" U-tetrad actually has a significantly worse solvation energy compared to its theoretical counterpart with a "standard" U-tetrad (Table 3). On the other hand, when it comes to stacking, the AMBER force field does not contain an explicit "base stacking" energy term and these interactions instead contribute to the electrostatic and van der Waals terms. Indeed, in our PBSA analysis both of these terms were significantly lower for the system with a "reversed" U-tetrad (Table 3), likely reflecting a more favorable base stacking in this conformation. In order to further confirm the better stacking interactions for the "reversed" U-tetrad, quantum mechanical (QM) energy calculations in a set of different geometries would be required (Lech et al. 2013). Nevertheless, our current MD and experimental results strongly point toward favorable base stacking interactions as an important driving force for chain reversal.

Stacking interactions and hydration can also be used to rationalize the unexpected behavior of $M 7$, the quadruplex containing pseudouridine. Introduction of pseudouridine not only does not interfere with any of the hydrogen bonding interactions in the "reversed" U-tetrad but additionally, it provides an opportunity for more such interactions (Supplemental Fig. S1). Unexpectedly, for M7 we observed a disordered 3'-terminal region and a significant drop in G-quadruplex stability. This result can be explained by a different charge distribution of $\Psi$ compared to the standard uridine, which must influence its preferred 
stacking geometries with other nucleobases. $\Psi$ is also wellknown to induce a very specific hydration pattern in its vicinity (Deb et al. 2019). Thus, the disappearance of the $3^{\prime}-$ terminal tetrad in $\mathrm{M} 7$ could be attributed to the poor stacking of $\Psi$ with the preceding $G$ residue in the "inverted" conformation and/or to the incompatibility of the hydration pattern around $\Psi$ with the solvent structure preferred by the "reversed" U-tetrad. The inability of the pseudouridine-modified G-quadruplex M7 to form a "reversed" Utetrad could also be related to the pseudouridine's preference for the C3'-endo sugar pucker (Davis 1995), as a C2'endo ribose conformation is observed in a typical "reversed" U-tetrad (Fig. 1). However, in contrast to an LNA modified residue, a pseudouridine could adapt other conformations depending on structural contexts (Davis 1995). Unfortunately we were unable to assess the $\Psi$ puckering state in the M7 G-quadruplex due to severe spectral overlap of the $\Psi 7-\mathrm{H}^{\prime}{ }^{\prime}$ proton with other resonances.

Another aspect to be discussed is the role of steric factors in the formation propensity of the "reversed" U-tetrad. Our results strongly suggest that the "reversed" U-tetrad is not able to tolerate any additional steric hindrance in its proximity. This is best exemplified by M4, the quadruplex containing $5 \mathrm{Me}-\mathrm{U}$, in which the presence of an additional methyl group on the $3^{\prime}$-terminal residue leads to the loss of a rigid structure, accompanied by an $\sim 18^{\circ} \mathrm{C}$ decrease in $\mathrm{T}_{\mathrm{m}}{ }^{*}$. The steric repulsion between the methyl groups and O4 atoms has actually long been appreciated and proposed to be the reason for the reduced stability of T-tetrads with respect to their U-counterparts $(\mathrm{Gu}$ and Leszczynski 2001). Just like in the case of M3, probably not the entire destabilization of M4 can be attributed to this effect, as other interactions-such as $2^{\prime} \mathrm{OH}$ hydrogen bonding - are also affected when the 3'-terminal residue becomes mobile. Another example is provided by $\mathrm{M} 2$, the quadruplex with 2'OMe modification. While this modification, just as in M1, was designed to selectively remove the $2^{\prime} \mathrm{OH}$ to phosphate hydrogen bonding, its structural effect is much more drastic involving the disappearance of the $3^{\prime}$-terminal $U$-tetrad accompanied by a $13^{\circ} \mathrm{C}-17^{\circ} \mathrm{C}$ $\left(\mathrm{K}^{+}\right.$vs. $\left.\mathrm{Na}^{+}\right)$drop in $\mathrm{T}_{\mathrm{m}}{ }^{*}$. This structural difference between $\mathrm{M} 1$ and $\mathrm{M} 2$ can be attributed to the difficulty to accommodate the bulky O-methyl group in the framework of the "reversed" U-tetrad conformation. Indeed, the NOE intensities and ${ }^{31} \mathrm{P}$ chemical shifts of $\mathrm{M} 2$ suggest that the fraction of "reversed" states in its conformational ensemble must be very limited.

An interesting example among the studied molecules is provided by quadruplex M5, featuring a dT residue at its 3'terminus. It displays a destabilization comparable to M4 (with a $5 \mathrm{Me}-\mathrm{U}$ residue). This appears to corroborate our earlier suggestion that any contributions of the $2^{\prime} \mathrm{OH}$ group of $5 \mathrm{Me}-\mathrm{U}$ to the quadruplex stability were already lost in M4 due to the conformational mobility caused by the presence of the methyl groups. Thus, it can be reiterated, with an in- creased level of certainty, that the quadruplex destabilization observed for M3 (3'-terminal cytidine) and M4 is not uniquely related to the hydrogen bonding in the tetrad, but rather to a whole array of different interactions being affected. The M6 quadruplex, featuring a ${ }^{\text {LNAT7 }}$ residue, behaves very similarly to M5, both in terms of structural preferences and thermal stability. With its melting temperature practically identical to that of M5 (dT), it constitutes a rare example where the inclusion of an LNA residue into a helical context does not lead to any increase of the thermal stability of the folded structure.

To check whether the 3'-terminal "reversed" U-tetrad can be reproduced in the context of a DNA G-quadruplex and used to imbue such a fold with additional stability, we have studied quadruplexes d(TGGTGGT) 4 , and $[d(T G G T G G) r(U)]_{4}$. While the melting experiments have revealed some moderate stabilization of $\mathrm{M} 10$ with respect to M9 (by $\sim 6.5^{\circ} \mathrm{C}$ ), this effect was much weaker than expected. The subsequent NMR analysis has revealed that while $\mathrm{U} 7$ in $\mathrm{M} 10$ is indeed involved in the formation of a rigid U-tetrad, this tetrad, however, forms without chain reversal. Thus, it appears that the "reversed" U-tetrad motif does not only need a tetramolecular G-quadruplex scaffold with a ribo- $U$ as the 3 '-terminal residue, but also requires this scaffold to assume A-form helical structure. It may be also noted that the $6.5^{\circ} \mathrm{C}$ difference in the melting temperature between $\mathrm{M} 9$ and $\mathrm{M} 10$ can be seen as another indirect estimate of the destabilizing effect that the presence of the methyl groups has on a T-tetrad.

\section{Interpretation of the $C D$ data}

We used CD spectroscopy to verify whether the presence of the "reversed" U-tetrad motif induces any characteristic features in the CD spectrum. The spectral diversity observed within the set of modified RNA G-quadruplexes turned out to be significantly higher than what might have been expected based on their NMR structural analysis. RNA G-quadruplexes M0 to M7 share the same core and differ only in the conformation of the $3^{\prime}$-terminal residue. Thus, all the differences observable between their CD spectra can be assigned directly to the state assumed by the 3'-terminal U-tetrad. Both the "standard" and "reversed" tetrad arrangements should provide some specific increments to the CD intensity, while the states with disordered, unstacked uridines are expected to provide negligible contributions. Both $\mathrm{M0}$ and $\mathrm{M} 1$ feature uniquely the "reversed" U-tetrad and their CD spectra are indeed almost perfectly superimposable. Their characteristic feature is a negative ellipticity maximum at $\sim 290 \mathrm{~nm}$. For all the other G-quadruplexes the exact populations of the "reversed," "standard," and "unstacked" states are not known. The somewhat different spectral shapes observed between M2-M7 likely reflect different populations of these states for each G-quadruplex. However, a common 
feature of all these spectra is the appearance of an additional, positive ellipticity maximum at $\sim 280 \mathrm{~nm}$. Figure $4 \mathrm{~B}$ and Supplemental Figure S10b present the difference in CD spectra between each modified G-quadruplex and MO. None of these residual profiles can be considered to represent purely the CD contribution of the "reversed" U-tetrad building block, due to the possible presence of varying amounts of the "standard" U-tetrad in modified $\mathrm{G}$-quadruplexes. However, there is a remarkable agreement regarding the spectral positions at which partial removal of the "reversed" U-tetrad induces the biggest changes. Namely, all the residuals but one (M2 in $\mathrm{K}^{+}$) have a marked negative ellipticity maximum at $\sim 285 \mathrm{~nm}$ and a comparable or slightly weaker positive maximum at $260 \mathrm{~nm}$. These observations provide the best approximation of the CD signature of the "reversed" U-tetrad motif in RNA achievable with the current data. This strongly suggests that this motif is indeed responsible for the negative ellipticity observed at $\sim 290 \mathrm{~nm}$ for M0, M1, and M8.

Finally, the surprising positive maximum at $\sim 280 \mathrm{~nm}$ observed for many of the modified G-quadruplexes is, in our opinion, related to the presence of the central U-tetrad. This is supported by the fact that the only CD spectrum with no trace of this band belonged to $\mathrm{M} 8$, for which the central U-tetrad was abolished. On the other hand, this maximum is also barely visible-only as a shoulder of the main band under $\mathrm{Na}^{+}$containing conditions-for two Gquadruplexes that do contain the central tetrad, $\mathrm{MO}$ and M1. For these molecules, the lack of a clear maximum at $\sim 280 \mathrm{~nm}$ is easily explained by the presence of a strong negative band in this region provided by the 3 '-terminal "reversed" U-tetrad (Fig. 4B).

An alternative explanation for the positive $C D$ maximum at $280 \mathrm{~nm}$ might be the formation of structures other than the parallel G-quadruplex at $C D$ concentrations. Especially, the presence of a hybrid $3+1 \mathrm{G}$-quadruplex could be postulated, as a CD maximum at $\sim 280 \mathrm{~nm}$ is very typical for such a fold. In order to check whether the sample dilution performed for CD spectroscopy could have provoked a transition of the parallel tetramolecular G-quadruplexes into other structures, a 1D-NMR spectrum was recorded for one of the diluted samples (M4) recovered after the CD measurements (Supplemental Fig. S12). The unchanged imino and aromatic proton regions in this spectrum ascertain that no structural rearrangements occur upon sample dilution and the G-quadruplex structures studied by $C D$ are the same as characterized by NMR above.

One more feature of the $C D$ spectra is the recurrent trend that for most of modified G-quadruplexes the positive maximum at $\sim 280 \mathrm{~nm}$ is stronger in the presence of $\mathrm{Na}^{+}$as compared to $\mathrm{K}^{+}$. While our current data is insufficient to unequivocally explain this observation, we believe that at least two different effects may contribute to it. Firstly, the stacking geometries may be slightly different in G-quadruplexes formed in the $\mathrm{Na}^{+}$and $\mathrm{K}^{+}$containing conditions, especially at the vicinity of the central U-tetrad that we propose to be responsible for the $C D$ band at $\sim 280$ $\mathrm{nm}$. For the nonmodified r(UGGUGGU) ${ }_{4} \mathrm{G}$-quadruplex the only significant chemical shift differences between the $\mathrm{Na}^{+}$ and $\mathrm{K}^{+}$containing conditions were observed for the imino protons of G3 and U4 (Andrałojć et al. 2019), suggesting that the type of ion may indeed influence the geometry of the central U-tetrad. Secondly, the populations of the different conformations assumed by the modified $3^{\prime}$-terminal residues may also differ between $\mathrm{Na}^{+}$and $\mathrm{K}^{+}$containing conditions. A somewhat lower population of the "reversed" state (that we link to the negative ellipticity at $\sim 290 \mathrm{~nm}$ ) in the $\mathrm{Na}^{+}$containing conditions would lead to a stronger positive peak $\sim 280 \mathrm{~nm}$.

\section{Conclusions}

In order to gain deeper insights into the origin of the exquisite stabilizing effect of the $3^{\prime}$-terminal U-tetrad, we studied the structures and thermal stabilities of a series of carefully designed 3'-modified G-quadruplexes. First of all, our results indicate a strict requirement for a nonmodified uridine residue for the stabilization to take place. Every chemical modification of this residue-even mutation to deoxy- $U$ that does not alter the G-quadruplex structure-led to at least a $10^{\circ} \mathrm{C}$ decrease in the apparent G-quadruplex melting temperature. As far as the specific interactions responsible for the stabilizing effect are concerned, we confirmed that both the $2^{\prime} \mathrm{OH}$ to phosphate hydrogen bonding, we have previously proposed (Andrałojć et al. 2019), and the hydrogen bonding within the tetrad have significant contributions. On the other hand, binding of additional $\mathrm{K}^{+}$ions in the $\mathrm{G}$-quadruplex groove seems to have a negligible effect on its thermal stability. The removal of the $2^{\prime} \mathrm{OH}$ to phosphate hydrogen bonding translates to a $10{ }^{\circ} \mathrm{C}$ drop in the apparent melting temperature. The hydrogen bonding within the tetrads appears to have an even greater significance (up to $22^{\circ} \mathrm{C}$ ), but it was not possible to quantify this contribution, due to the structural changes that occur when this interaction is removed. However, we also found that neither of these two interactions can be considered to be the primary incentive for the chain reversal between the last two residues, as the removal of the $2^{\prime} \mathrm{OH}$ interaction does not abolish the "reversed" conformation, while the $\mathrm{H}$ bonding between the bases can be easily achieved both with and without chain reversal. The results we obtained for a G-quadruplex with a $3^{\prime}$-terminal $\Psi$ and through MD simulations suggest that preferential base stacking interactions achievable in the "reversed" conformation may be the primary cause for chain reversal. Regarding the chemical requirements for the formation of the "reversed" U-tetrad, our study reveals the crucial role of steric factors. The "reversed" U-tetrad motif appears to be tightly packed to the point in which the introduction of any additional 
functional group to the participating residue precludes its formation. Consequently, a thymidine base, for example, was unable to stably assume the "reversed" conformation in any context we studied (RNA G-quadruplex, DNA Gquadruplex, ribose sugar, deoxyribose sugar). On top of that, our experiments with DNA G-quadruplexes have shown the structural requirements for the formation of the "reversed" U-tetrad to be even more stringent, as the motif did not form in a DNA context even when a ribouridine residue was placed at the 3 '-terminus. Additionally, we were also able to correlate the presence of a "reversed" U-tetrad in a RNA G-quadruplex with the appearance of a CD minimum at $\sim 290 \mathrm{~nm}$.

Overall, our results provide detailed insights into the energetics of the "reversed" U-tetrad motif and the requirements for its formation. The now well-understood 3'terminal "reversed" U-tetrad motif can be thought of as a general building block that can be introduced into RNA G-quadruplexes to stabilize the parallel-stranded tetramolecular fold. As such, the motif may find its use in nucleic acid nanotechnology to provide tetramolecular Gquadruplex folds with additional stability or even to push a G-rich RNA sequence toward such a fold over other types of secondary structures it may be prone to assume.

\section{MATERIALS AND METHODS}

\section{RNA sample preparation}

All oligoribonucleotides were obtained using standard phosphoramidite chemistry and purified as described in our previous work (Andrałojć et al. 2019). In order to prevent quadruplex dimerization, each oligoribonucleotide was synthetized harboring an additional 5'-phosphate group.

After purification each oligoribonucleotide was divided between two buffering conditions: (i) $150 \mathrm{mM} \mathrm{NaCl}, 10 \mathrm{mM}$ $\mathrm{Na}_{2} \mathrm{HPO}_{4} / \mathrm{NaH}_{2} \mathrm{PO}_{4}, 0.1 \mathrm{mM}$ EDTA, $\mathrm{pH} 6.8$ (referred to as "sodium buffer" from now on); and (ii) $50 \mathrm{mM} \mathrm{KCl}, 10 \mathrm{mM}$ $\mathrm{K}_{2} \mathrm{HPO}_{4} / \mathrm{KH}_{2} \mathrm{PO}_{4}, 0.1 \mathrm{mM}$ EDTA, pH 6.8 ("potassium buffer"). Centrifugation on Amicon filters was used to wash away any unwanted salts remaining after the oligomer purification phase. Both samples were annealed by heating at $90^{\circ} \mathrm{C}$ for $5 \mathrm{~min}$ and then slowly cooled down to the room temperature and stored at $4^{\circ} \mathrm{C}$. The slow association of tetramolecular G-quadruplexes was monitored by NMR spectroscopy until each sample reached a steady state (usually after several weeks).

\section{NMR spectroscopy}

All NMR spectra were collected using a $700 \mathrm{MHz}$ Bruker Avance III spectrometer equipped with a $\mathrm{QCl}$ CryoProbe. The resonance assignment of nonexchangeable protons and phosphorus atoms was achieved using standard procedures (Varani et al. 1996; Wijmenga and van Buuren 1998), through the analysis of NOESY, TOCSY, HC-HSOC, and HP-COSY spectra recorded in $100 \% \mathrm{D}_{2} \mathrm{O}$ at $25^{\circ} \mathrm{C}$. The exchangeable protons were assigned us- ing NOESY spectra measured in $90 \% \mathrm{H}_{2} \mathrm{O} / 10 \% \mathrm{D}_{2} \mathrm{O}$ at $5^{\circ} \mathrm{C}$ and $25^{\circ} \mathrm{C}$. The proton chemical shifts were referenced using TSP while the ${ }^{31} \mathrm{P}$ chemical shifts were indirectly referenced using proton chemical shifts, using the ${ }^{1} \mathrm{H} /{ }^{31} \mathrm{P}$ frequency ratio of 0.404808636 . All the spectra were analyzed in NMRFAM-Sparky (Lee et al. 2015). The assigned chemical shifts of nonexchangeable protons were deposited to the BMRB under entry IDs: 50242 and 50244 to 50249.

\section{UV melting}

Thermal denaturation experiments using a JASCO V-750 spectrophotometer with a water-cooled thermoprogrammer were recorded at 260 and $295 \mathrm{~nm}$ using $5 \mathrm{~mm}(150 \mu \mathrm{L})$ quartz microcuvettes. The samples were protected against evaporation by the silicone oil. The temperature range was $5^{\circ} \mathrm{C}-92^{\circ} \mathrm{C}$, using the scan rate $0.1^{\circ} \mathrm{C} / \mathrm{min}$. Oligonucleotides were melted in both the sodium and potassium containing buffers (see above). All melting curves were collected for samples previously confirmed by NMR to contain predominantly the well-folded quadruplexes (see below), after proper dilution. Melting curves were analyzed with the program OriginPro8.

\section{MD simulations}

The dynamics of the r(UGGUGGU) ${ }_{4}$ G-quadruplex were simulated starting from two very distinct initial conformations: $(A)$ its native state with a "reversed" U-tetrad (taken from PDBID: 6GE1); and (B) an alternative state in which the U7 residue forms a "standard" U-tetrad. This alternative conformation was obtained by imposing the geometry adapted by the G3-U4 dinucleotide step in 6GE1 onto the G6-U7 step, followed by a short restrained MD for relaxation. Both systems were then subjected to 500-nsec-long molecular dynamics runs. All MD simulations were performed using the parm99bsc0 $\chi_{\text {OL3 } 3}$ force field (Cheatham et al. 1999; Pérez et al. 2007; Zgarbová et al. 2011) with the SPC/E water model and Joung-Cheatham parameters for monovalent cations (Joung and Cheatham 2008). For both the $A$ and $B$ systems, five $\mathrm{K}^{+}$ions were manually added into the quadruplex channel. Quadruplexes were solvated using an octahedral box of SPC/E water with a minimum distance between box walls and a solute of $10 \AA$, and additional $\mathrm{K}^{+}$ions were added to neutralize the system. The solvated systems were minimized and equilibrated. The long-range electrostatics were calculated using the particle mesh Ewald method with the nonbonded cutoff set to $9 \AA$. The covalent bonds were constrained using SHAKE, and the integration time step was set to 2 fsec. The Langevin thermostat with collision frequency 2.0 psec $^{-1}$ was used to control the temperature and Berendsen barostat for constant pressure simulation.

Each obtained trajectory was than analyzed using the MMPBSA and MM-GBSA methods (Miller et al. 2012). The MMPBSA python script included in AMBER18 was used for these calculations. Snapshots for analysis were taken at $100 \mathrm{psec}$ intervals over the period 100-500 nsec MD trajectories. The channel cations $\mathrm{K}^{+}$were explicitly included in the free-energy calculations. The sizes of the $\mathrm{K}^{+}$ions were modified to $1.705 \AA$ (following Islam et al. 2016). The entropy contributions were not included in the energy analyses. The average energies obtained were 
decomposed into contributions from different components of the force field, similarly to the approach used in Srinivasan et al. (1998).

\section{CD spectroscopy}

The circular dichroism spectra (CD) were recorded using a JASCO J815 spectropolarimeter equipped with a Peltier temperature controller. Cuvettes with a path length of $1 \mathrm{~cm}$ were used (sample volumes $2500 \mu \mathrm{L}$ ). Spectra were collected in the range between 214 and $340 \mathrm{~nm}$, as a sum of three repetitions at $25^{\circ} \mathrm{C}$ and the buffer baseline was subtracted from each spectrum. $C D$ spectra were expressed in the units of molar ellipticity $\Delta \varepsilon\left(\mathrm{cm}^{2} \mathrm{mmol}^{-1}\right)$, without normalization by the number of residues in the molecule.

\section{SUPPLEMENTAL MATERIAL}

Supplemental material is available for this article.

\section{ACKNOWLEDGMENTS}

The calculations were performed at Poznań Supercomputing and Networking Center. We thank the European Center for Bioinformatics and Genomics (ECBiG), Poznan, Poland, for computer laboratory support.

Received May 28, 2020; accepted September 17, 2020.

\section{REFERENCES}

Andrałojć W, Małgowska M, Sarzyńska J, Pasternak K, Szpotkowski K, Kierzek R, Gdaniec Z. 2019. Unraveling the structural basis for the exceptional stability of RNA G-quadruplexes capped by a uridine tetrad at the $3^{\prime}$ terminus. RNA 25: 121-134. doi:10.1261/rna .068163 .118

Berman HM, Westbrook J, Feng Z, Gilliland G, Bhat TN, Weissig H, Shindyalov IN, Bourne PE. 2000. The Protein Data Bank. Nucleic Acids Res 28: 235-242. doi:10.1093/nar/28.1.235

Cáceres C, Wright G, Gouyette C, Parkinson G, Subirana JA. 2004. A thymine tetrad in d(TGGGGT) quadruplexes stabilized with $\mathrm{Tl}^{+} /$ $\mathrm{Na}^{+}$ions. Nucleic Acids Res 32: 1097-1102. doi:10.1093/nar/ gkh269

Cheatham TE, Cieplak P, Kollman PA. 1999. A modified version of the Cornell et al. force field with improved sugar pucker phases and helical repeat. J Biomol Struct Dyn 16: 845-862. doi:10.1080/ 07391102.1999.10508297

Cheong C, Moore PB. 1992. Solution structure of an unusually stable RNA tetraplex containing G- and U-quartet structures. Biochemistry 31: 8406-8414. doi:10.1021/bi00151a003

Creze C, Rinaldi B, Haser R, Bouvet P, Gouet P. 2007. Structure of a d (TGGGGT) quadruplex crystallized in the presence of $\mathrm{Li}^{+}$ions. Acta Crystallogr Sect D Biol Crystallogr 63: 682-688. doi:10.1107/ S0907444907013315

Davis DR. 1995. Stabilization of RNA stacking by pseudouridine. Nucleic Acids Res 23: 5020-5026. doi:10.1093/nar/23.24.5020

Deb I, Popenda Ł, Sarzyńska J, Małgowska M, Lahiri A, Gdaniec Z, Kierzek R. 2019. Computational and NMR studies of RNA duplexes with an internal pseudouridine-adenosine base pair. Sci Rep 9: 113. doi:10.1038/s41598-018-37186-2 del Villar-Guerra R, Trent JO, Chaires JB. 2018. G-quadruplex secondary structure obtained from circular dichroism spectroscopy. Angew Chemie Int Ed 57: 7171-7175. doi:10.1002/anie .201709184

Deng J, Xiong Y, Sundaralingam M. 2001. X-ray analysis of an RNA tetraplex (UGGGGU) 4 with divalent $\mathrm{Sr}^{2+}$ ions at subatomic resolution (0.61 A). Proc Natl Acad Sci 98: 13665-13670. doi:10.1073/pnas .241374798

Duchardt-Ferner E, Wöhnert J. 2017. NMR experiments for the rapid identification of $\mathrm{P}=\mathrm{OH}-\mathrm{X}$ type hydrogen bonds in nucleic acids. J Biomol NMR 69: 101-110. doi:10.1007/s10858-0170140-7

Duchardt-Ferner E, Ferner J, Wöhnert J. 2011. Rapid identification of noncanonical RNA structure elements by direct detection of $\mathrm{OH}$... $\mathrm{O} P, \mathrm{NH} \ldots \mathrm{OP}$, and $\mathrm{NH} 2$... O P hydrogen bonds in solution NMR spectroscopy. Angew Chem Int Ed Engl 50: 7927-7930. doi:10 .1002/anie.201101743

Dvorkin SA, Karsisiotis Al, da Silva MW. 2018. Encoding canonical DNA quadruplex structure. Sci Adv 4: eaat3007. doi:10.1126/ sciadv.aat3007

Fyfe AC, Dunten PW, Martick MM, Scott WG. 2015. Structural variations and solvent structure of $r($ GGGGU) quadruplexes stabilized by $\mathrm{Sr}^{2+}$ ions. J Mol Biol 427: 2205-2219. doi:10.1016/j.jmb.2015 .03 .022

Gorenstein DG. 1984. Phosphorus-31 chemical shifts: principles and empirical observations. In Phosphorous-31 NMR (ed. Gorenstein DG), pp. 7-36, Elsevier.

Gu J, Leszczynski J. 2001. A theoretical study of thymine and uracil tetrads: structures, properties, and interactions with the monovalent $\mathrm{K}^{+}$cation. J Phys Chem A 105: 10366-10371. doi:10.1021/ jp004550v

Islam B, Stadlbauer P, Neidle S, Haider S, Sponer J. 2016. Can we execute reliable MM-PBSA free energy computations of relative stabilities of different guanine quadruplex folds? J Phys Chem B 120: 2899-2912. doi:10.1021/acs.jpcb.6b01059

Joung IS, Cheatham TE. 2008. Determination of alkali and halide monovalent ion parameters for use in explicitly solvated biomolecular simulations. J Phys Chem B 112: 9020-9041. doi:10.1021/ jp8001614

Kwok CK, Merrick CJ. 2017. G-quadruplexes: prediction, characterization, and biological application. Trends Biotechnol 35: $997-$ 1013. doi:10.1016/j.tibtech.2017.06.012

Laughlan G, Murchie Al, Norman DG, Moore MH, Moody PC, Lilley DM, Luisi B. 1994. The high-resolution crystal structure of a parallel-stranded guanine tetraplex. Science 265: 520-524. doi:10.1126/science.8036494

Lech CJ, Heddi B, Phan AT. 2013. Guanine base stacking in G-quadruplex nucleic acids. Nucleic Acids Res 41: 2034-2046. doi:10 $.1093 /$ nar/gks1110

Lee MPH, Parkinson GN, Hazel P, Neidle S. 2007. Observation of the coexistence of sodium and calcium ions in a DNA G-quadruplex ion channel. J Am Chem Soc 129: 10106-10107. doi:10.1021/ ja0740869

Lee W, Tonelli M, Markley JL. 2015. NMRFAM-SPARKY: enhanced software for biomolecular NMR spectroscopy. Bioinformatics 31: 1325-1327. doi:10.1093/bioinformatics/btu830

Mendoza O, Porrini M, Salgado GF, Gabelica V, Mergny J-L. 2015. Orienting tetramolecular G-quadruplex formation: the quest for the elusive RNA antiparallel quadruplex. Chem A Eur J 21: 6732-6739. doi:10.1002/chem.201500358

Miller BR, McGee TD, Swails JM, Homeyer N, Gohlke H, Roitberg AE. 2012. MMPBSA.py: an efficient program for end-state free energy calculations. J Chem Theory Comput 8: 3314-3321. doi:10.1021/ ct300418h 
Neidle S. 2017. Quadruplex nucleic acids as targets for anticancer therapeutics. Nat Rev Chem 1: 0041. doi:10.1038/s41570-017-0041

Pan B, Xiong Y, Shi K, Deng J, Sundaralingam M. 2003a. Crystal structure of an RNA purine-rich tetraplex containing adenine tetrads: implications for specific binding in RNA tetraplexes. Structure 11: 815-823. doi:10.1016/S0969-2126(03)00107-2

Pan B, Xiong Y, Shi K, Sundaralingam M. 2003b. An eight-stranded helical fragment in RNA crystal structure: implications for tetraplex interaction. Structure 11: 825-831. doi:10.1016/S0969-2126(03) 00108-4

Pan B, Xiong Y, Shi K, Sundaralingam M. 2003c. Crystal structure of a bulged RNA tetraplex at 1.1 Å resolution: implications for a novel binding site in RNA tetraplex. Structure 11: 1423-1430. doi:10 .1016/j.str.2003.09.017

Pan B, Shi K, Sundaralingam M. 2006a. Base-tetrad swapping results in dimerization of RNA quadruplexes: implications for formation of the i-motif RNA octaplex. Proc Natl Acad Sci 103: 3130-3134. doi:10.1073/pnas.0507730103

Pan B, Shi K, Sundaralingam M. 2006b. Crystal structure of an RNA quadruplex containing inosine tetrad: implications for the roles of $\mathrm{NH}_{2}$ group in purine tetrads. $\mathrm{J} \mathrm{Mol} \mathrm{Biol} \mathrm{363:} \mathrm{451-459.} \mathrm{doi:10}$ $.1016 / j . j m b .2006 .08 .022$

Patel P, Hosur RV. 1999. NMR observation of T-tetrads in a parallel stranded DNA quadruplex formed by Saccharomyces cerevisiae telomere repeats. Nucleic Acids Res 27: 2457-2464. doi:10 $.1093 /$ nar/27.12.2457

Pérez A, Marchán I, Svozil D, Sponer J, Cheatham TE, Laughton CA, Orozco M. 2007. Refinement of the AMBER force field for nucleic acids: improving the description of $\alpha / \gamma$ conformers. Biophys $J$ 92: 3817-3829. doi:10.1529/biophysj.106.097782

Popenda M, Miskiewicz J, Sarzynska J, Zok T, Szachniuk M. 2019. Topology-based classification of tetrads and quadruplex structures. Bioinformatics 36: 1129-1134. doi:10.1093/bioinfor matics/btz738
Rhodes D, Lipps HJ. 2015. G-quadruplexes and their regulatory roles in biology. Nucleic Acids Res 43: 8627-8637. doi:10.1093/nar/ gkv862

Srinivasan J, Cheatham TE, Cieplak P, Kollman PA, Case DA. 1998. Continuum solvent studies of the stability of DNA, RNA, and phosphoramidate-DNA helices. J Am Chem Soc 120: 9401-9409. doi:10.1021/ja981844+

Varani G, Aboul-ela F, Allain FH-T. 1996. NMR investigation of RNA structure. Prog Nucl Magn Reson Spectrosc 29: 51-127. doi:10 .1016/0079-6565(96)01028-X

Webba da Silva M. 2007. Geometric formalism for DNA quadruplex folding. Chem A Eur J 13: 9738-9745. doi:10.1002/chem .200701255

Wijmenga SS, van Buuren BNM. 1998. The use of NMR methods for conformational studies of nucleic acids. Prog Nucl Magn Reson Spectrosc 32: 287-387. doi:10.1016/S0079-6565(97) 00023-X

Xiao CD, Shibata T, Yamamoto Y, Xu Y. 2018. An intramolecular antiparallel G-quadruplex formed by human telomere RNA. Chem Commun 54: 3944-3946. doi:10.1039/C8CC01427B

Xu Y, Ishizuka T, Kimura T, Komiyama M. 2010. A U-tetrad stabilizes human telomeric RNA G-quadruplex structure. J Am Chem Soc 132: 7231-7233. doi:10.1021/ja909708a

Zgarbová M, Otyepka M, Šponer J, Mládek A, Banáš P, Cheatham TE, Jurečka P. 2011. Refinement of the Cornell et al. nucleic acids force field based on reference quantum chemical calculations of glycosidic torsion profiles. J Chem Theory Comput 7: 28862902. doi:10.1021/ct200162x

Zhou J, Amrane S, Rosu F, Salgado GF, Bian Y, Tateishi-Karimata H, Largy E, Korkut DN, Bourdoncle A, Miyoshi D, et al. 2017. Unexpected position-dependent effects of ribose G-quartets in G-quadruplexes. J Am Chem Soc 139: 7768-7779. doi:10.1021/ jacs. 7 b00648 

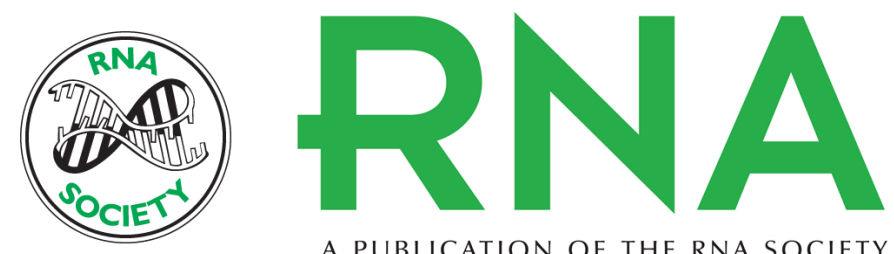

A PUBLICATION OF THE RNA SOCIETY

\section{The origin of the high stability of 3'-terminal uridine tetrads: contributions of hydrogen bonding, stacking interactions, and steric factors evaluated using modified oligonucleotide analogs}

Witold Andralojc, Karol Pasternak, Joanna Sarzynska, et al.

RNA 2020 26: 2000-2016 originally published online September 23, 2020

Access the most recent version at doi:10.1261/rna.076539.120

Supplemental Material

References

Creative Commons License

Email Alerting Service
http://rnajournal.cshlp.org/content/suppl/2020/09/23/rna.076539.120.DC1

This article cites 42 articles, 5 of which can be accessed free at: http://rnajournal.cshlp.org/content/26/12/2000.full.html\#ref-list-1

This article is distributed exclusively by the RNA Society for the first 12 months after the full-issue publication date (see http://rnajournal.cshlp.org/site/misc/terms.xhtml). After 12 months, it is available under a Creative Commons License (Attribution-NonCommercial 4.0 International), as described at http://creativecommons.org/licenses/by-nc/4.0/.

Receive free email alerts when new articles cite this article - sign up in the box at the top right corner of the article or click here.

To subscribe to $R N A$ go to:

http://rnajournal.cshlp.org/subscriptions 